\title{
AXIOMATIC PRIMARY AND TERTIARY DECOMPOSITION THEORY
}

\author{
BY \\ JOHN A. RILEY
}

Introduction. M. Auslander has given, in unpublished notes, an axiomatic treatment of the classical primary decomposition theory for modules over commutative Noetherian rings. The purpose of this paper is to show that this axiom scheme may be suitably abstracted and modified so as to include also the recent Lesieur-Croisot theory [1-3] of tertiary decomposition in modules over arbitrary (i.e., not necessarily commutative) Noetherian rings. We do this by developing an abstract theory of what we call decomposition functors, having both the primary and tertiary theories as special cases.

Briefly, if $\Lambda$ is any ring, a decomposition functor $\Gamma$ assigns to a pair $(M, N)$, consisting of a $\Lambda$-module $M$ and submodule $N$, a collection $\Gamma(M, N)$ of ideals in $\Lambda$. This assignment is subject to several axioms which, a posteriori at least, can be considered as embodying the essential features of the primary and tertiary decomposition theories. In $\S \S 1$ and 2 we give the axioms for these decomposition functors and develop some of their basic properties. The material in these two sections is, apart from its more general content, a rearrangement and elaboration of the axioms given by Auslander.

In $\$ 3$ we summmarize the basic facts concerning the primary and tertiary theories, and show how these may be defined within the framework of decomposition functors.

Returning to the abstract theory, $\S 4$ presents certain results concerning the comparison of two decomposition functors. In $\$ 5$ we define the concept of normal decomposition functors; the primary and tertiary theories are examples of such decompositions. Putting together the results of $\S \S 4$ and 5 we obtain the following remarkable result: for finitely generated modules over a Noetherian ring, any two normal decomposition theories coincide, i.e., assign the same set $\Gamma(M, N)$ of ideals to a pair $(M, N)$. Using the fact that the tertiary theory is normal, we conclude that for finitely generated modules over a Noetherian ring, the tertiary theory is the unique normal decomposition theory. In this sense the tertiary decomposition theory is the only natural generalization, to arbitrary Noetherian rings, of the classical primary decomposition theory in commutative rings. This result answers a question of Goldie's [4, p. 127], and underlines the potential importance of the tertiary theory for the study of Noetherian rings.

Presented to the Society, September 11, 1961; received by the editors January 2, 1961. 
In $\$ 6$ we give a certain generalization of the notion of primary submodule. Here we adopt a somewhat novel point of view: we consider that the property of being primary is not an absolute property of a submodule, but rather is a property which a submodule may or may not posses relative to a given decomposition theory. Thus, if $\Gamma$ is a decomposition functor, we say that a submodule $N$ of $M$ is a $\Gamma$-primary submodule of $M$ if, first of all, $N$ is a $\Gamma$-submodule of $M$ in the sense that $\Gamma(M, N)$ consists of a single ideal, say $\Gamma(M, N)=\{\mathfrak{a}\}$, and secondly, if $\mathfrak{a}^{k} M \subseteq N$ for some $k \geqq 0$. In the commutative Noetherian case, the $\Gamma$-primary submodules are exactly the usual primary submodules; in fact, in this case the three concepts, $\Gamma$-submodule, $\Gamma$-primary submodule, and primary submodule, coincide. In general, if all $\Gamma$-submodules are $\Gamma$-primary, we say that $\Gamma$ is a primary or a classical, decomposition theory. We give an example to show that the tertiary theory is not, in general, a primary decomposition theory. In this connection we prove the following extension of a result of Lesieur-Croisot: a normal decomposition functor is primary if and only if $\Lambda$ has the Artin-Rees property relative to $\Gamma$ (see $\S 6$ for the meaning). Application to the case of finitely generated modules over a Noetherian ring $\Lambda$ yields: $\Lambda$ has a primary decomposition theory if and only if it has the Artin-Rees property. This is another solution to a problem first posed by Murdoch [5] and solved by Tominaga [6].

The application of this result to commutative Noetherian rings yields a purely "ideal-theoretic" proof of the so-called Artin-Rees theorem.

We round off $\S 6$ by showing that the Artin rings with a primary decomposition theory are just the direct sums of primary rings. This indicates that, from one point of view, the Artin-Rees property is a direct generalization of commutativity.

Finally, in $\$ 7$ we consider briefly several results and examples concerning what we call classical rings, i.e., Noetherian rings with a primary decomposition theory.

Two notable examples of classical rings are finitely generated separable $R$ algebras, where $R$ is a commutative, Noetherian ring, and maximal orders over Dedekind rings.

Throughout the paper all rings will be assumed to possess a unit element. A Noetherian ring means one with the ascending chain condition on left ideals; and all modules are unitary, left modules.

I am indebted to Professor Maurice Auslander of Brandeis University, and to the staff of the Parke Mathematical Laboratories, particularly to Dr. Lorenzo Calabi, for their kind encouragement and assistance in the preparation of this paper.

1. Admissible categories. Decomposition functions. Let $\Lambda$ be a ring. A pair $\left(M, M^{\prime}\right)$ is a $\Lambda$-module $M$, together with a submodule $M^{\prime}$. An admissible map, $f:\left(M, M^{\prime}\right) \rightarrow\left(N, N^{\prime}\right)$ is a monomorphism $0 \rightarrow M / M^{\prime} \rightarrow{ }^{f} N / N^{\prime}$. If $M^{\prime}=(0)$, we will write simply " $M$ "' instead of “ $(M,(0))$." An admissible map $(M,(0))$ 
$\rightarrow^{f}(N,(0))$ will be written simply as $0 \rightarrow M \rightarrow^{f} N$. A collection $C$ of pairs and admissible maps is called an admissible category for decomposition theory if the following axioms hold:

(1) if $\left(M, M^{\prime}\right) \in C$, then $M$ and $M^{\prime}$ are in $C$ (i.e., the pairs $(M,(0)),\left(M^{\prime},(0)\right)$ are in $C$ ), together with the inclusion map $0 \rightarrow M^{\prime} \rightarrow M$;

(2) if $M \in C$, and $0 \rightarrow M^{\prime} \rightarrow^{f} M$ is a monomorphism, then $\left(M, M^{\prime}\right)$ and the $\operatorname{map} f$ are in $C$;

(3) if $\left(M, M^{\prime}\right) \in C$, then $M / M^{\prime}=\left(M / M^{\prime},(0)\right)$ is in $C$, and we identify $M / M^{\prime}$ with the pair $\left(M, M^{\prime}\right)$ by the obvious map;

(4) if $\left(M, M^{\prime}\right)$ and $\left(N, N^{\prime}\right)$ are in $C$, then so are $\left(M \oplus N, M^{\prime} \oplus N^{\prime}\right)$ and the inclusions maps $0 \rightarrow M \rightarrow M \oplus N, 0 \rightarrow N \rightarrow M \oplus N$.

Axioms (1)-(3) imply that we could just as well consider a category, $C^{\prime}$, of modules satisfying the single requirement that if $M \in C^{\prime}$, and $0 \rightarrow M^{\prime} \rightarrow M \rightarrow M^{\prime \prime}$ $\rightarrow 0$ is exact, then $M^{\prime}$ and $M^{\prime \prime}$ are in $C^{\prime}$. We prefer to retain the terminology of "pairs" as being suggestive of the objects with which decomposition theory is concerned.

Examples of admissible categories are: the category of all pairs $\left(M, M^{\prime}\right)$ and monomorphisms $0 \rightarrow M^{\prime} \rightarrow M$; and, if $\Lambda$ is a Noetherian ring, the category of all pairs $\left(M, M^{\prime}\right)$ of finitely generated $\Lambda$-modules and monomorphisms $0 \rightarrow M^{\prime} \rightarrow M$.

In the sequel we will use (1)-(4) for the most part without explicit mention $\left({ }^{1}\right)$.

Let $C$ be an admissible category, and $\Gamma$ a covariant functor from $C$ to a category $A$ of sets of ideals in $\Lambda$ (with the set theoretic inclusions as maps). We say that $\Gamma$ is a predecomposition theory for $C$ (relative to $A$ ) if it satisfies the following conditions:

I. if $\left(M, M^{\prime}\right) \in C$, and $M$ is the union of a totally ordered family $\left\{M_{\alpha}\right\}$ of submodules containing $M^{\prime}$, then $\Gamma\left(M, M^{\prime}\right)=\bigcup_{\alpha} \Gamma\left(M_{\alpha}, M^{\prime}\right)$;

II. if $M \in C$, and $0 \rightarrow M^{\prime} \rightarrow M \rightarrow M^{\prime \prime} \rightarrow 0$ is exact, then $\Gamma\left(M^{\prime}\right) \subseteq \Gamma(M) \subseteq \Gamma\left(M^{\prime}\right)$ $\cup \Gamma\left(M^{\prime \prime}\right)$;

III. $\Gamma(M, N)=\varnothing$ if and only if $M=N$.

For convenience, we will write $\Gamma(M / N)$ instead of $\Gamma(M, N)$. This notation is consistent with the identification of $(M, N)$ and $M / N$.

Since $\Gamma$ is covariant, we have $\Gamma\left(M / M^{\prime}\right) \subset \Gamma\left(N / N^{\prime}\right)$ whenever there is an admissible map $0 \rightarrow M / M^{\prime} \rightarrow N / N^{\prime}$. It follows from (II) that

$$
\Gamma\left(M / M^{\prime} \oplus N / N^{\prime}\right)=\Gamma\left(M / M^{\prime}\right) \cup \Gamma\left(N / N^{\prime}\right),
$$

whenever $\left(M, M^{\prime}\right)$ and $\left(N, N^{\prime}\right)$ are in $C$.

(1) The necessity for introducing such formal considerations as "admissible category" and "admissible map" lies only in the desire to have a more or less precise description of the domain of definition of the decomposition theories which we discuss. A potential advantage of this generality is that the theory is not restricted a priori to a particular situation, for example, to the case of finitely generated modules. 
Let $(M, N) \in C$. We say that $N$ is a $\Gamma$-submodule of $M$ if $\Gamma(M / N)$ consists of a single element. It is clear that $N$ is a $\Gamma$-submodule of $M$ if and only if (0) is a $\Gamma$ submodule of $M / N$. If $M^{\prime} / N$ is a submodule of $M / N$ then $M^{\prime} / N$ is a $\Gamma$-submodule of $M / N$ if and only if (0) is a $\Gamma$-submodule of $M / N / M^{\prime} / N=M / M^{\prime}$, i.e., if and only if $M^{\prime}$ is a $\Gamma$-submodule of $M$.

We say that a finite set $M_{1}, \ldots, M_{k}$ of submodules of $M$ is a $\Gamma$-decomposition of $N$ in $M$ if:

(a) the $M_{i}$ are $\Gamma$-submodules of $M$;

(b) $M_{1} \cap \ldots \cap M_{k}=N$, and no proper subset of the $M_{i}$ has $N$ for its intersection;

(c) for $i \neq j, \Gamma\left(M / M_{i}\right) \neq \Gamma\left(M / M_{j}\right)$.

It is not difficult to verify, using the remarks above, that $\left\{M_{1}, \ldots, M_{k}\right\}$ is a $\Gamma$-decomposition of $N$ in $M$ if and only if the collection $\left\{M_{1} / N, \ldots, M_{k} / N\right\}$ is a $\Gamma$-decomposition of $(0)$ in $M / N$.

Note that a finite collection of submodules $M_{i}$ satisfying (a) and (c) is a $\Gamma$ decomposition of $N$ in $M$ if and only if the map $\phi: M / N \rightarrow M / M_{1} \oplus \ldots \oplus M / M_{k}$ defined by $\phi(M+N)=\left(f_{1}(M), \ldots, f_{k}(M)\right)$, where $f_{i}$ is the canonical map $f_{i}: M$ $\rightarrow M / M_{i}$, is a monomorphism, but does not remain a monomorphism if some of the $M / M_{i}$ are dropped.

An element $(M, N)$ of $C$ is said to be $\Gamma$-finite if $\Gamma(M / N)$ is a finite set.

Proposition 1.1. Let $(M, N)$ be $\Gamma$-finite, with $\Gamma(M / N)=\left\{\mathfrak{a}_{1}, \ldots, \mathfrak{a}_{k}\right\}$, and suppose that $M_{1}, \ldots, M_{k}$ are $\Gamma$-submodules of $M$ such that $\Gamma\left(M / M_{i}\right)=\left\{\mathfrak{a}_{i}\right\}$. Then $\left\{M_{1}, \ldots, M_{k}\right\}$ is a $\Gamma$-decomposition of $N$ in $M$ if and only if $M_{1} \cap \ldots \cap M_{k}$ $=N$.

Proof. If $\left\{M_{1}, \ldots, M_{k}\right\}$ is a $\Gamma$-decomposition of $N$ in $M$, then $M_{1} \cap \ldots \cap M_{k}=N$. To prove the converse we need only show that no proper subset of the $M_{i}$ has $N$ for its intersection. Suppose that $M_{1} \cap \ldots \cap M_{r}=N$, with $r<k$. Then the map $\phi: M / N \rightarrow M / M_{1} \oplus \ldots \oplus M / M_{r}$ described above is a monomorphism. Hence $\Gamma(M / N) \subseteq \Gamma\left(M / M_{1} \oplus \ldots \oplus M / M_{r}\right)=\Gamma\left(M / M_{1}\right) \cup \ldots \cup \Gamma\left(M / M_{r}\right)$ $=\left\{\mathfrak{a}_{1}, \ldots, \mathfrak{a}_{r}\right\}$. This contradicts the fact that $\Gamma(M / N)=\left\{\mathfrak{a}_{1}, \ldots, \mathfrak{a}_{k}\right\}$. Thus no proper subset of the $M_{i}$ has $N$ for its intersection, and the proposition is proved.

Proposition 1.2. Let $\left\{M_{1}, \ldots, M_{k}\right\}$ be a $\Gamma$-decomposition of $N$ in $M$. Let $\Gamma\left(M / M_{i}\right)=\left\{\mathfrak{a}_{i}\right\}, i=1,2, \ldots, k$. Then:

(a) $\Gamma(M / N)=\left\{\mathfrak{a}_{1}, \ldots, \mathfrak{a}_{k}\right\}$;

(b) $\Gamma\left(M_{i} / N\right)=\left\{\mathfrak{a}_{1}, \ldots, \hat{\mathfrak{a}}_{i}, \ldots, \mathfrak{a}_{k}\right\}$, where the circumflex means that $\mathfrak{a}_{i}$ has been omitted; and

(c) $\Gamma\left(\left[M_{1} \cap \ldots \cap \hat{M}_{i} \cap \ldots \cap M_{k}\right] / N\right)=\left\{\mathfrak{a}_{i}\right\}$.

Proof. Set $N_{i}=M / M_{i}$. We have a monomorphism $M / N \rightarrow N_{1} \oplus \ldots \oplus N_{k}$, and so $\Gamma(M / N) \subseteq \Gamma\left(N_{1} \oplus \ldots \oplus N_{k}\right)=\left\{\mathfrak{a}_{1}, \ldots, \mathfrak{a}_{k}\right\}$. The map $M / N \rightarrow N_{j}$ induces 
a map $g_{j}:\left[M_{1} \cap \ldots \cap \hat{M}_{j} \cap \ldots \cap M_{k}\right] / N \rightarrow N_{j}$. Since $M_{1} \cap \ldots \cap M_{k}=N, g_{j}$ 1s a monomorphism. Since $M_{1} \cap \ldots \cap \hat{M}_{j} \cap \ldots \cap M_{k} \neq N$, we have $\varnothing \neq$ $\Gamma\left(\left[M_{1} \cap \ldots \cap \hat{M}_{j} \cap \ldots \cap M_{k}\right] / N\right) \subseteq \Gamma\left(N_{j}\right)=\left\{\mathfrak{a}_{j}\right\}$. Thus

$$
\Gamma\left(M_{1} \cap \ldots \cap \hat{M}_{j} \cap \ldots \cap M_{k} / N\right)=\left\{\mathfrak{a}_{j}\right\},
$$

proving (c). Since $M_{1} \cap \ldots \cap \hat{M}_{j} \cap \ldots \cap M_{k} / N \subseteq M / N$, we have $\mathfrak{a}_{j} \in \Gamma(M / N)$. Hence $\Gamma(M / N)=\left\{\mathfrak{a}_{1}, \ldots, \mathfrak{a}_{k}\right\}$, proving (a). Finally, consider the exact sequence $0 \rightarrow M_{i} / N \rightarrow M / N \rightarrow N_{i} \rightarrow 0$. We have $\Gamma(M / N) \subseteq \Gamma\left(M_{i} / N\right) \cup \Gamma\left(N_{i}\right)=\Gamma\left(M_{i} / N\right) \cup\left\{a_{i}\right\}$. Thus $\left\{\mathfrak{a}_{1}, \ldots, \hat{\mathfrak{a}}_{i}, \ldots, \mathfrak{a}_{k}\right\} \subseteq \Gamma\left(M_{\imath} / N\right)$. On the other hand, we have a monomorphism $0 \rightarrow M_{i} / N \rightarrow N_{1} \oplus \ldots \oplus \hat{N}_{i} \oplus \ldots \oplus N_{k}$ so that $\Gamma\left(M_{i} / N\right) \subseteq \Gamma\left(N_{1} \oplus \ldots \oplus \hat{N}_{i}\right.$ $\left.\oplus \ldots \oplus N_{k}\right)=\left\{\mathfrak{a}_{1}, \ldots, \hat{\mathfrak{a}}_{i}, \ldots, \mathfrak{a}_{k}\right\}$. Hence $\Gamma\left(M_{i} / N\right)\left\{\mathfrak{a}_{1}, \ldots, \hat{\mathfrak{a}}_{i}, \ldots, \mathfrak{a}_{k}\right\}$, and the proposition is proved.

The following sequence of results, obtained for the case of the tertiary theory Lesieur-Croisot [2], is concerned with the 'relative' situation of a chain $N^{\prime} \subset N \subset M$ of submodules. The study of these relative decompositions is the key to the connection between ideal theory and the Artin-Rees theorem.

LEMmA 1.3. Let $R, S$ be submodules of $M$ such that $N=R \cap S$, with $(M, N) \in C$. If $R$ is a $\Gamma$-submodule of $M$, then $N$ is a $\Gamma$-submodule of $S$, and $\Gamma(S / N)=\Gamma(M / R)$.

Proof. We have an obvious monomorphism $0 \rightarrow S / N \rightarrow M / R$. Thus $\Gamma(S / N)$ $\subseteq \Gamma(M / R)$. If $R$ is a $\Gamma$-submodule of $M$, then $\Gamma(M / R)$ consists of a single element, say a. Then $\Gamma(S / N)=\{\mathfrak{a}\}$ also, so that $N$ is a $\Gamma$-submodule of $S$.

LEMMA 1.4. Suppose that $N$ has a $\Gamma$-decomposition in $M$, and that $S / N$ is a nontrivial submodule of $M / N$. If $N$ is a $\Gamma$-submodule of $S$, then there is a $\Gamma$-submodule $R$ of $M$ such that $R \cap S=N$, and $\Gamma(M / R)=\Gamma(S / N)$.

Proof. Let $M_{1}, \ldots, M_{k}$ be a $\Gamma$-decomposition of $N$ in $M$. Let $\Gamma\left(M / M_{i}\right)=\left\{\mathfrak{a}_{i}\right\}$, $i=1, \ldots, k$. Set $N_{i}=M_{i} \cap S$. Then the $N_{i}$ are $\Gamma$-submodules of $S$ by the preceding lemma, and $\Gamma\left(S / N_{i}\right)=\Gamma\left(M / M_{i}\right)=\left\{\mathfrak{a}_{i}\right\}$. Now $N_{1} \cap \ldots \cap N_{k}=N$, and by dropping suitable $N_{i}$, we may obtain a subset, say $N_{1}, \ldots, N_{r}$, such that $N_{1} \cap \ldots \cap N_{r}$ $=N$, and such that no proper subset of the $N_{1}, \ldots, N_{r}$ has $N$ for its intersection. Thus we obtain a $\Gamma$-decomposition of $N$ in $S$. Since by assumption, $N$ is a $\Gamma$ submodule of $S$, it follows that $r=1$, and hence that $N=M_{1} \cap S$. We take $R=M_{1}$, and the lemma is proved.

Combining these two lemmas we have:

Proposition 1.5. Let $N$ have a $\Gamma$-decomposition in $M$, and let $S / N$ be a nontrivial submodule of $M / N$. A necessary and sufficient condition that $N$ be a $\Gamma$-submodule of $S$ is that there exist a $\Gamma$-submodule $R$ of $M$ such that $R \cap S=N$. In this case $\Gamma(S / N)=\Gamma(M / R)$.

Propositon 1.6. Let $N$ have a $\Gamma$-decomposition in $M$ and suppose that $S$ is a submodule of $M$ containing $N$. Then there exists a submodule $R$ of $M$ such that $N=R \cap S$ with $\Gamma(S / N)=\Gamma(M / R)$. 
Proof. If $S=N$, we take $R=M$. Suppose that $S \neq N$. Let $M_{1}, \ldots, M_{k}$ be a $\Gamma$-decomposition of $N$ in $M$. By the method used in the proof of 1.4 above, we obtain a $\Gamma$-decomposition $N_{1}, \ldots, N_{r}$ of $N$ in $S$ in the form $N_{i}=M_{i} \cap S$, $i=1, \ldots, r$. We have $\Gamma\left(S / N_{i}\right)=\Gamma\left(M / M_{i}\right)=\left\{\mathfrak{a}_{i}\right\}$, say, and $\Gamma(S / N)=\left\{\mathfrak{a}_{1}, \ldots, \mathfrak{a}_{r}\right\}$. Let $R=M_{1} \cap \ldots \cap M_{r}$. The $M_{i} / R$ are a $\Gamma$-decomposition of $(0)$ in $M / R$, with $\Gamma\left(M / R / M_{i} / R\right)=\Gamma\left(M / M_{i}\right)=\left\{\mathfrak{a}_{i}\right\}$. Thus $\Gamma(M / R)=\left\{\mathfrak{a}_{1}, \ldots, \mathfrak{a}_{r}\right\}=\Gamma(S)$. Evidently $N=R \cap S$, and the proposition is proved.

2. Existence of $\Gamma$-decompositions. From Proposition 1.2 we see that a necessary condition for $N$ to have a $\Gamma$-decomposition in $M$ is that $(M, N)$ be $\Gamma$-finite. If, for each $\Gamma$-finite pair $(M, N) \in C, N$ has a $\Gamma$-decomposition in $M$ we say that $\Gamma$ is a decomposition theory for $C$ (relative to the given category $A$ of ideals).

In order to obtain sufficient conditions for $\Gamma$ to be a decomposition theory, we consider the property:

(P): For each $a \in \Gamma(M / N)$, there is a submodule $M^{\prime}$ of $M$ containing $N$ such that $\Gamma\left(M^{\prime} / M\right)=\{\mathfrak{a}\}$.

Proposition 2.1. Let $\Gamma$ be a predecomposition theory on $C$, and suppose that $\Gamma$ satisfies (P). Then $\Gamma$ is a decomposition theory on $C$.

The proof requires a lemma.

Lemma 2.2 Assume that $\Gamma$ has property $(\mathrm{P})$. Let $(M, N) \in C, M \neq N$. Suppose that $\Gamma(M / N)=P \cup Q$, where $P, Q$ are disjoint nonempty subsets of $\Gamma(M / N)$. If $N^{\prime} / N$ is a proper submodule of $M / N$ such that $\Gamma\left(N^{\prime} / N\right) \subset P$, then there is a submodule $M^{\prime}$ of $M$ which contains $N^{\prime}$, such that $\Gamma\left(M^{\prime} / N\right)=P, \Gamma\left(M / M^{\prime}\right)=Q$.

Proof. It suffices to assume that $N=(0)$. Then, let $F$ denote the family of all submodules $M^{\prime}$ of $M$ such that $\Gamma\left(M^{\prime}\right) \subseteq P$.

Since $\Gamma\left(\bigcup M_{i}\right)=\bigcup \Gamma\left(M_{i}\right)$ for a totally ordered family $M_{i}$ of submodules of $M$, it follows, using Zorn's lemma that $F$ has maximal elements. Let $M^{\prime}$ be one of them. Since $\Gamma\left(M^{\prime}\right) \subseteq P$, and $P \neq \Gamma(M)$, we see that $M^{\prime} \neq M$, so that $M / M^{\prime}=M^{\prime \prime}$ is a nonzero element of $C$. Now we observe that in order to prove the lemma, it is sufficient to show that $\Gamma\left(M^{\prime \prime}\right) \subseteq Q$. For suppose that $\Gamma\left(M^{\prime \prime}\right) \subseteq Q$. Then $P \cup Q=\Gamma(M) \subseteq \Gamma\left(M^{\prime}\right) \cup \Gamma\left(M^{\prime \prime}\right) \subseteq P \cup Q$, and then it follows that $\Gamma\left(M^{\prime}\right) \cup \Gamma\left(M^{\prime \prime}\right)=P \cup Q$. Since $P \cap Q=\varnothing$, and since $\Gamma\left(M^{\prime}\right) \subseteq P, \Gamma\left(M^{\prime \prime}\right) \subseteq Q$, we obtain $\Gamma\left(M^{\prime}\right)=P$, and $\Gamma\left(M^{\prime \prime}\right)=Q$, the desired conclusion. Thus, to show that $\Gamma\left(M^{\prime \prime}\right) \subseteq Q$, let $a \in \Gamma\left(M^{\prime \prime}\right)$. By (P), there is a submodule $N^{\prime \prime} / M^{\prime}$ of $M^{\prime \prime}=M / M^{\prime}$ such that $\Gamma\left(N^{\prime \prime} / M^{\prime}\right)=\{\mathfrak{a}\}$. Then from the sequence $0 \rightarrow M^{\prime} \rightarrow N^{\prime \prime} \rightarrow N^{\prime \prime} / M^{\prime} \rightarrow 0$ we obtain:

$$
\Gamma\left(N^{\prime \prime}\right) \subseteq \Gamma\left(M^{\prime}\right) \cup \Gamma\left(N^{\prime \prime} / M^{\prime}\right)=\Gamma\left(M^{\prime}\right) \cup\{\mathfrak{a}\} .
$$

If $\mathfrak{a} \notin Q$, then $\Gamma\left(N^{\prime \prime}\right) \subseteq P$, since $\Gamma\left(N^{\prime \prime}\right) \subseteq P \cup Q(=\Gamma(M))$ and $P$ and $Q$ are dis- 
joint. But then the existence of this $N^{\prime \prime}$ contradicts the maximality of $M^{\prime}$. Thus $a \in Q$, and $\Gamma\left(M^{\prime \prime}\right) \subseteq Q$. The lemma is proved.

Proof of 2.1. Again, it suffices to take $N=(0)$. Let $M$ be a $\Gamma$-finite element of $C$, with $\Gamma(M)=\left\{\mathfrak{a}_{1}, \ldots, \mathfrak{a}_{k}\right\}$. By property $(\mathrm{P})$, there is a submodule $N_{1}$ of $M$ such that $\Gamma\left(N_{1}\right)=\left\{a_{1}\right\}$. By the lemma, there is, for each $i=2,3, \ldots, k$, a submodule $M_{i}$ of $M$ containing $N_{1}$ such that:

(a) $M / M_{i} \in C$;

(b) $\Gamma\left(M_{i}\right)=\left\{\mathfrak{a}_{1}, \ldots, \hat{\mathfrak{a}}_{i}, \ldots, \mathfrak{a}_{k}\right\}$, where the circumflex means "omit $\mathfrak{a}_{i}$ "; and

(c) $\Gamma\left(M / M_{i}\right)=\left\{\mathfrak{a}_{i}\right\}$.

Again, corresponding to $\mathfrak{a}_{2}$, there is a submodule $N_{2}$ of $M$ such that $\Gamma\left(N_{2}\right)=\left\{\mathfrak{a}_{2}\right\}$. Then there is, again by the lemma, a submodule $M_{1}$ of $M$, containing $N_{1}$, such that $\Gamma\left(M_{1}\right)=\left\{a_{2}, \ldots, a_{k}\right\}, \Gamma\left(M / M_{1}\right)=\left\{a_{1}\right\}$. Thus we have constructed a family $\left\{M_{1}, \ldots, M_{k}\right\}$ of submodules of $M$ such that:

(1) $M_{i}$ is a $\Gamma$-submodule of $M$, for $i=1,2, \ldots, k$;

(2) $\Gamma\left(M / M_{i}\right)=\left\{\mathfrak{a}_{i}\right\} ; i=1, \ldots, k$.

Now $M_{1} \cap \ldots \cap M_{k}$ is a submodule of each $M_{i}$, so that $\Gamma\left(M_{1} \cap \ldots \cap M_{k}\right) \subseteq \Gamma\left(M_{i}\right)$ for $i=1, \ldots, k$ and so $\Gamma\left(M_{1} \cap \ldots \cap M_{k}\right) \subseteq \Gamma\left(M_{1}\right) \cap \ldots \cap \Gamma\left(M_{k}\right)$. This latter intersection is empty, by (6) above. Hence $\Gamma\left(M_{1} \cap \ldots \cap M_{k}\right)=\emptyset$ and so $M_{1} \cap \ldots$ $\cap M_{k}=(0)$. Applying Proposition 1.1, we see that the set $\left\{M_{1}, \ldots, M_{k}\right\}$ is a $\Gamma$-decomposition of $(0)$ in $M$. Hence for any $\Gamma$-finite element $M \in C$, (0) has a $\Gamma$-decomposition in $M$, and $\Gamma$ is a decomposition theory for $C$.

I do not know whether the converse of 2.1 is generally true, i.e., whether the existence of a $\Gamma$-decomposition theory for $C$ necessarily implies that $\Gamma$ has property (P). However, if the elements of $C$ are $\Gamma$-finite, property $(\mathrm{P})$ follows immediately by (c) of Proposition 1.2. Thus,

COROLlaRY 2.3. Let $\Gamma$ be a predecomposition theory on $C$. If the elements of $C$ are $\Gamma$-finite, then $\Gamma$ is a decomposition theory for $C$ if and only if $\Gamma$ satisfies (P).

COROLlary 2.4. Assume that $\Lambda$ is a Noetherian ring, and that the predecomposition theory $\Gamma$ satisfies property $(\mathrm{P})$. Then if $(M, N) \in C$, with $M$ finitely generated, $(M, N)$ is $\Gamma$-finite.

Proof. It suffices to assume that $N=(0)$. Suppose that $\Gamma(M)$ is not finite. Since $M$ is Noetherian, the family of $\Gamma$-finite submodules, $S$, has maximal elements (this family is not empty, by (P)). Let $S$ be one of these maximal elements. Let $a \in \Gamma(M), \mathfrak{a} \notin \Gamma(S)$. By Lemma 2.2 , there is a submodule $M^{\prime}$ of $M$ such that $S \subseteq M^{\prime}$, and $\Gamma\left(M^{\prime}\right)=\Gamma(S) \cup\{\mathfrak{a}\}$. Thus $M^{\prime}$ properly contains $S$ and is $\Gamma$-finite. This contradicts the maximality of $S$, and proves that $\Gamma(M)$ is finite. Combining these last two results, we have:

COROLlaRY 2.5. Let $\Lambda$ be a Noetherian ring and $C$ an admissible category 
of pairs of finitely generated $\Lambda$-modules. Then $\Gamma$ is a decomposition theory on $C$ if and only if $\Gamma$ satisfies property $(\mathrm{P})$.

We close this section with an example of a predecomposition theory which is not a decomposition theory.

Let $\Lambda$ be a commutative, Noetherian ring, and $M$ a finitely generated $\Lambda$-module. The support, Supp $M$, of $M$ is defined in [7, p. 20] as the collection of those primes $\mathfrak{p}$ in $\Lambda$ for which $M_{\mathfrak{p}} \neq 0$. It is quite easy to verify that the functor $(M, N) \rightarrow \operatorname{Supp} M / N$ is a predecomposition theory on the category of pairs of finitely generated modules. Now in [7] it is shown that Supp $M$ is precisely the set of primes which contain the annihilator of $M$. Using this fact we will see in $\$ 7$ that Supp is a decomposition functor if and only if $\Lambda$ is an Artin ring. In fact we will prove more generally that in any Noetherian ring, $\Lambda$, the functor which assigns to a pair $(M, N)$ of finitely generated $\Lambda$-modules those primes which contain the annihilator of $M / N$ is a decomposition theory if and only if $\Lambda$ is, modulo its radical, a finite direct sum of simple rings.

3. The primary and tertiary theories. Let $\Lambda$ be a ring, and $M$ a $\Lambda$-module. An element $x$ of $\Lambda$ is said to be an annihilating element for $M$ if $x N=(0)$ for some nonzero submodule $N$ of $M$. A (left) ideal $\mathfrak{a}$ in $\Lambda$ is an annihilating (left) ideal for $M$ if $a N=(0)$ for some nonzero submodule $N$ of $M$. We will see in $\S 5$ that if $\Lambda$ is Noetherian, and $M$ is finitely generated, then a left ideal in $\Lambda$ is an annihilating left ideal for $M$ if and only if it consists entirely of annihilating elements. The annihilator, Ann $M$, of the $\Lambda$-module $M$, is the set $\{x \in \Lambda \mid x M=(0)\}$. It is clear that Ann $M$ is a two-sided ideal in $\Lambda$.

A (two-sided) ideal $\mathfrak{p}$ in $\Lambda$ is said to be a prime ideal if $\mathfrak{I m} \subseteq \mathfrak{p}$ implies either $\mathfrak{l} \subseteq \mathfrak{p}$ or $\mathfrak{m} \subseteq \mathfrak{p}$, where $\mathfrak{l}, \mathfrak{m}$ are left ideals in $\Lambda$.

Proposition 3.1. Suppose that $\Lambda$ is a Noetherian ring, and that $M$ is a nonzero $\Lambda$-module. Then the family of annihilating left ideals for $M$ has maximal elements. If $\mathfrak{P}$ is one of these, then $\mathfrak{P}$ is a two-sided, prime ideal, and there is a nontrivial submodule $N$ of $M$ such that $\mathfrak{P}=\operatorname{Ann} N^{\prime}$ for all nontrivial submodules $N^{\prime}$ of $N$. Finally, if $M$ is finitely generated, the set of maximal annihilating ideals for $M$ is a finite set.

Proof. That the family of annihilating left ideals has maximal elements is clear, since $\Lambda$ is Noetherian. Let $\mathfrak{P}$ be such a maximal element, and let $\mathfrak{P N}=(0)$ for some nontrivial submodule $N$ of $M$. Then $\mathfrak{P} \subseteq$ Ann $N$, and by the maximality of $\mathfrak{P}, \mathfrak{P}=\operatorname{Ann} N$. Thus $\mathfrak{P}$ is a two-sided ideal. Let $N^{\prime}$ be a nontrivial submodule of $N$. Then $\mathfrak{P} N^{\prime}=(0)$ so that $\mathfrak{P} \subseteq$ Ann $N^{\prime}$. Again, by the maximality of $\mathfrak{P}$, $\mathfrak{P}=$ Ann $N^{\prime}$. Now let $\mathbb{I m} \subseteq \mathfrak{P} ; \mathfrak{l}, \mathfrak{m}$ two left ideals in $\Lambda$.

Then Im $N=(0)$. If $\mathfrak{m} N=(0)$, then $\mathfrak{m} \subseteq$ Ann $N=\mathfrak{P}$. If $\mathfrak{m} N \neq(0)$, then $\mathfrak{l} \subseteq$ Ann $m N$ $=\mathfrak{P}$, and $\mathfrak{l} \subseteq \mathfrak{P}$. Thus either $\mathfrak{l}$ or $\mathfrak{m}$ is contained in $\mathfrak{P}$ and $\mathfrak{P}$ is a prime ideal. That the set of maximal annihilating ideals for $M$ is a finite set when $M$ is finitely 
generated can be deduced immediately from the fact that it is a subset of $T(M)$, where $T$ is the tertiary decomposition functor discussed below in this section. However, the following direct proof is interesting. Denote by $H$ the set of maximal annihilating ideals for $M$. Let $\mathfrak{P}_{1}, \mathfrak{P}_{2}, \ldots$ be a sequence of elements of $H$, and let $\mathfrak{P}_{i}=$ Ann $M_{i}, \mathrm{i}=1,2, \ldots$. Then consider the ascending chain $M_{1} \subseteq M_{1}+M_{2}$ $\subseteq M_{1}+M_{2}+M_{3} \subseteq \ldots$. Since $M$ is Noetherian, this chain becomes stationary, so that there is an $n$ such that $M_{1}+\ldots+M_{n}=M_{1}+\ldots+M_{n+p}$ for all $p \geqq 0$. Then $\mathfrak{P}_{1} \cap \ldots \cap \mathfrak{P}_{n}=\operatorname{Ann}\left(M_{1}+\ldots+M_{n}\right)=\operatorname{Ann}\left(M_{1}+\ldots+M_{n+p}\right)=\mathfrak{P}_{1} \cap$ $\ldots \cap \mathfrak{P}_{n+p}$ for all $p \geqq 0$. Thus for all $k>n, \mathfrak{P}_{k} \supseteq \mathfrak{P}_{1} \cap \ldots \cap \mathfrak{P}_{n}$, and hence, since $\mathfrak{P}_{k}$ is prime, $\mathfrak{P}_{k} \supseteq \mathfrak{P}_{i}$ for some index $i$ between 1 and $n$. Then by the maximality of $\mathfrak{P}_{i}$, we have $\mathfrak{P}_{k}=\mathfrak{P}_{i}$. This shows that any sequence of elements of $H$ contains only finitely many distinct elements, and it follows that $H$ is a finite set.

If $\mathfrak{a}$ is an ideal in $\Lambda$, we denote by rad $\mathfrak{a}$ the radical of $\mathfrak{a}$, i.e., the intersection of the prime ideals containing $\mathfrak{a}$. It is well known that each element of $\operatorname{rad} \mathfrak{a}$ is nilpotent modulo $a$. If $\Lambda$ is a Noetherian ring, we can conclude further $\left({ }^{2}\right)$ that $\operatorname{rad} \mathfrak{a}$ itself is nilpotent modulo $\mathfrak{a}$, i.e., $(\operatorname{rad} \mathfrak{a})^{h} \subseteq \mathfrak{a}$ for some $h$. If $N$ is a submodule of $M$, we define the radical, $\operatorname{rad} M / N$, of $N$ in $M$, to be $\operatorname{rad} M / N=$ $\operatorname{rad}$ Ann $M / N$. We say that $N$ is a primary submodule of $M$ if the annihilating elements for $M / N$ are contained in $\operatorname{rad} M / N$. This is a direct generalization of the concept of primary submodule in the commutative case, and specializes to this latter concept if $\Lambda$ is a commutative ring. If $N$ is a submodule of $M$, we define the tertiary radical, ter $M / N$, of $N$ in $M$ to be the set of elements of $\Lambda$ which are annihilating elements for all nontrivial submodules of $M / N$. It is shown in [1, Théorème 1.1] that ter $M / N$ is an ideal in $\Lambda$, and that if $\Lambda$ is commutative, ter $M / N=\operatorname{rad} M / N$. A submodule $N$ is said to be a tertiary submodule of $M$ if the annihilating elements for $M / N$ are contained in ter $M / N$. It is easy to see that $\operatorname{rad} M / N \subseteq \operatorname{ter} M / N$, using the fact that the elements $\operatorname{rad} M / N$ are nilpotent modulo Ann $M / N$; hence primary submodules are also tertiary submodules. If $\Lambda$ is a commutative ring, tertiary submodules, conversely, are primary; if $\Lambda$ is not commutative, nonprimary tertiary submodules may exist. An example of this situation is given at the end of this section.

If $N$ is a tertiary submodule of $M$, then ter $M / N$ is a prime ideal in $\Lambda$; this is proved in [1]. Since primary submodules are tertiary, this shows that the radical of a primary submodule is also a prime ideal, a fact well known in the commutative case.

A submodule $N$ of $M$ is said to be irreducible if it may not be written as the intersection of two proper submodules of $M$, i.e., if $N=N_{1} \cap N_{2} \Rightarrow$ either $N_{1}=N$ or $N_{2}=N$. It is well known that if $M$ is Noetherian, each submodule of $M$ may be written as a finite intersection of irreducible submodules.

(2) By using Levitzki's theorem: a nil ideal in a Noetherian ring is nilpotent. Cf. [8, Theorem 1, p. 199]. 
The basic facts concerning the tertiary decomposition theory can now be summarized. The proofs may be found in the papers of Lesieur and Croisot to which we have already referred. These results are also, of course, consequences of the axiomatic treatment, to be given presently, of the tertiary decomposition functor.

Thus, let $\Lambda$ be a Noetherian ring and $M$ a finitely generated $\Lambda$-module. Then the irreducible submodules of $M$ are tertiary submodules, and any submodule $N$ of $M$ may be written as a finite intersection $N=N_{1} \cap \ldots \cap N_{k}$ of tertiary submodules of $M$. Further, the decomposition may be chosen to be (i) irredundant, in the sense that no proper subset of the $N_{1}, \ldots, N_{k}$ has $N$ for its intersection, and (ii) reduced, in the sense that for $N_{i} \neq N_{j}$, ter $M / N_{i} \neq \operatorname{ter} M / N_{j}$. The tertiary radicals, ter $M / N_{i}$, of the $N_{i}$, are prime ideals, and their totality is an invariant of $N$, in the sense that if $N=N_{1}^{\prime} \cap \ldots \cap N_{l}^{\prime}$ is another irredundant, reduced decomposition of $N$ into tertiary submodules, then the set $\left\{\operatorname{ter} M / N_{i}^{\prime} \mid i=1, \ldots, l\right\}$ coincides with the set $\left\{\operatorname{ter} M / N_{i} \mid i=1, \ldots, k\right\}$. It follows immediately that $l=k$, so that the length, $k$, of the decomposition is also an invariant of $N$. The primes occurring as tertiary radicals of the 'components' in an irredundant, reduced decomposition of $N$ are called the primes belonging to $N$ (in $M$ ). Lesieur has shown [3, Théorème 2] that $\mathfrak{p}$ is a prime belonging to $N$ in $M$ if and only if $\mathfrak{p}$ is an essential residual of $M / N$, i.e., if and only if there is a nontrivial submodule $\bar{N}$ of $M / N$ such that $\mathfrak{p}$ is the annihilator of all nontrivial submodules of $\bar{N}$. It follows that $N$ is a tertiary submodule of $M$ if and only if the set of primes belonging to $N$ in $M$ consists of exactly one element.

If $\Lambda$ is a commutative Noetherian ring, we have mentioned that the tertiary submodules coincide with the primary ones. The above list can then be translated into the basic facts of the usual primary decomposition theory by simply replacing "tertiary" by "primary," "ter" by "rad," etc. Note that the primes belonging to $N$ in $M$ receive a simpler characterization: they are simply those primes which are the annihilators of nonzero elements of $M / N$. This is shown by the following computation. Let $\mathfrak{p}$ be a prime belonging to $N$ in $M$; i.e., $\mathfrak{p}$ is the annihilator of all nontrivial submodules of some nontrivial submodule $\bar{N}$ of $M / N$. Let $x \in \bar{N}$. Then $\mathfrak{p}=\operatorname{Ann} \Lambda x=\operatorname{Ann} x$. Conversely, if $\mathfrak{p}=\operatorname{Ann} x$, set $\bar{N}=\Lambda x$, and let $S$ be a nontrivial submodule of $\bar{N}$. Then $S=\mathfrak{b} x$ for some ideal $\mathfrak{b}$ in $\Lambda$, and we have $\mathfrak{p} S=\mathfrak{p b} x=\mathfrak{b} \mathfrak{p} x=(0)$, so that $\mathfrak{p} \subseteq$ Ann $S$. On the other hand, if $\mathfrak{a} S=0$, then $\mathfrak{a} \mathfrak{b} x=0$, and so $\mathfrak{a} \mathfrak{b} \subseteq \mathfrak{p}$. Since $\mathfrak{b} x \neq(0), \mathfrak{b} \neq \mathfrak{p}$, and thus since $\mathfrak{p}$ is prime, $\mathfrak{a} \subseteq \mathfrak{p}$. Hence Ann $\mathfrak{b} x \subseteq \mathfrak{p}$, and we conclude that $\mathfrak{p}=\operatorname{Ann} S$. Thus $\mathfrak{p}$ is the annihilator of all nontrivial submodules of $\Lambda x$, and hence $\mathfrak{p}$ belongs to $N$ in $M$.

Now we give the axiomatic development of the tertiary theory.

Let $\Lambda$ be a Noetherian ring, and $C$ an admissible category for decomposition theory. If $(M, N) \in C$, we define $T(M, N)$ to be the set of all ideals $\mathfrak{p}$ in $\Lambda$ which 
have the following property: there is a nontrivial submodule $\bar{N}$ of $M / N$ such that $\mathfrak{p}$ is the annihilator of all nontrivial submodules of $\bar{N}$. T is obviously a covariant functor from $C$ to the category of sets of ideals in $\Lambda$. We remark that the elements of $T(M / N)$ are prime ideals (for $\mathfrak{p} \in T(M / N)$, let $\mathfrak{a} \mathfrak{b} \subseteq \mathfrak{p}, \mathfrak{b} \notin \mathfrak{p}$. Let $\mathfrak{p}=\operatorname{Ann} N^{\prime}$ for all nonzero submodules $N^{\prime}$ of $\bar{N}$. Then $\mathfrak{a} \mathfrak{b} \bar{N}=(0), \mathfrak{b} \bar{N} \neq(0)$, so that $\mathfrak{a} \subseteq \operatorname{Ann} \mathfrak{b} \bar{N}=\mathfrak{p}$ ).

Proposition 3.1 shows that if $M \neq(0)$, the maximal annihilating ideals for $M / N$ are elements of $T(M / N)$; thus $T(M / N)=\varnothing$ if and only if $M=N$. It is also clear that if $M=\bigcup_{\alpha} M_{\alpha}$ is the union of a totally ordered family of submodules $M_{\alpha}$, each containing $N$, then $T(M / N)=\bigcup T\left(M_{\alpha} / N\right)$. In order to show that $T$ is a predecomposition theory on $C$, we have only to prove:

LEMMA 3.2. If $0 \rightarrow M^{\prime} \rightarrow M \rightarrow M^{\prime \prime} \rightarrow 0$ is an exact sequence, with $M \in C$, then $T\left(M^{\prime}\right) \subseteq T(M) \subseteq T\left(M^{\prime}\right) \cup T\left(M^{\prime \prime}\right)$.

Proof. It suffices to prove that if $\mathfrak{a} \in T(M), \mathfrak{a} \notin T\left(M^{\prime}\right)$, then $\mathfrak{a} \in T\left(M^{\prime \prime}\right)$. Let $\mathfrak{a} \in T(M), \mathfrak{a} \notin T\left(M^{\prime}\right)$. There is a submodule $N$ of $M$ such that $\mathfrak{a}=\operatorname{Ann} N^{\prime}$ for all nontrivial submodules $N^{\prime}$ of $N$. Suppose that $N \cap M^{\prime} \neq(0)$. Then $N \cap M^{\prime}$ is a nontrivial submodule of $N$ so that $a=\operatorname{Ann} N^{\prime \prime}$ for all submodules $N^{\prime \prime} \neq(0)$ of $N \cap M^{\prime}$. But since $N \cap M^{\prime}$ is also a nontrivial submodule of $M^{\prime}$, this gives $\mathfrak{a} \in T\left(M^{\prime}\right)$, contrary to our assumption. Hence $N \cap M^{\prime}=(0)$, and $N$ is isomorphic to a submodule of $M^{\prime \prime}$. Hence $a \in T\left(M^{\prime \prime}\right)$.

We conclude that $T$ is a predecomposition theory on $C$. It is immediate from the definition that $T$ satisfies property (P), and is therefore a decomposition theory on $C$. Further, the elements of $T(M / N)$ are annihilating ideals for $M / N$, and contain the annihilator, Ann $M / N$, of $M / N$, so that $T$ is normal, according to the definition of $\S 5$. Thus $T$ is a normal decomposition theory on $C$.

Corollary 3.3. Assume that $C$ is an admissible category of pairs of finitely generated modules. Then the decomposition theory $T$ coincides with the LesieurCroisot theory of tertiary decomposition.

Proof. If $M \in C$, we have seen that the primes belonging to (0) in $M$ in the tertiary theory are exactly the primes possessing the property used above to define the elements of $T(M)$. Further, a submodule $N$ of $M$ is tertiary if and only if there is a unique prime belonging to it. It follows that the elements of $T(M)$ are the primes belonging to (0) in $M$, so that the $T$-submodules of $M$ are exactly the tertiary submodules of $M$, and, finally that the tertiary decompositions of $(0)$ in $M$ are exactly the $T$-decompositions of (0) in $M$.

In view of this result, we will call the decomposition theory $T$ the tertiary theory on $C$. The $T$-submodules will be called tertiary submodules.

If $\Lambda$ is a commutative ring, the tertiary theory for finitely generated modules reduces to the classical primary decomposition theory. In this case we may define 
$T(M / N)$ to be the set of primes which are the annihilators of nonzero elements of $M / N\left({ }^{3}\right)$.

Apropos the primary theory, we could define, for any, not necessarily commutative, Noetherian ring $\Lambda$, a predecomposition theory $\mathscr{P}$ by the same formula: $\mathscr{P}(M / N)$ is the set of those primes in $\Lambda$ which are the annihilators of nontrivial cyclic submodules of $M / N$. I have not been able to determine whether or not $\mathscr{P}$ is a decomposition functor on the category of pairs of finitely generated modules (equivalently, whether $\mathscr{P}$ verifies property (P)); if it is, then of course, in view of the uniqueness theorem of $\S 5$, it will coincide with the tertiary theory.

We close this section with an example showing that the tertiary theory does not always coincide with the primary theory, i.e., that tertiary submodules are not always primary submodules.

Let $Q$ denote the field of rational numbers, and define an algebra $\Lambda$ over $Q$ by: $e_{1}^{2}=e_{1}, e_{2}^{2}=e_{2}, n^{2}=0 ; e_{1} e_{2}=e_{2} e_{1}=0 ; e_{1} n=n e_{2}=n ; n e_{1}=e_{2} n=0$. Then $\Lambda$ is the algebra of linear combinations $a_{1} e_{1}+a_{2} e_{2}+a_{3} n ; a_{i} \in Q$. It is a finite dimensional algebra over $Q$, and is thus an Artin, and hence a Noetherian, ring. The unit element is $e_{1}+e_{2}$. By straightforward computation we find that: (1) $\Lambda e_{1}$ $=Q e_{1} ; \Lambda n=Q n$, and $\Lambda e_{2}=Q e_{2}+Q n ; \quad$ (2) the ideals $m_{1}=\Lambda e_{1}+\Lambda n$ and $\mathrm{m}_{2}=\Lambda e_{2}$ are the only maximal (two-sided) ideals in $\Lambda$, and thus, since $\Lambda$ is an Artin ring, the only nonzero primes in $\Lambda$; (3) $\mathfrak{m}_{2} \mathfrak{m}_{1}=(0)$, so that $\mathfrak{m}_{2}$ is an annihilating ideal for $\Lambda / \Lambda e_{1}$; it follows by the maximality of $m_{2}$ that $T\left(\Lambda / \Lambda e_{1}\right)=\left\{m_{2}\right\}$; (4) the radical of $\Lambda / \Lambda e_{1}$ is $\Lambda n=m_{1} \cap m_{2}$, and $m_{2}$ is an annihilating ideal for $\Lambda / \Lambda e_{1}$ which is not contained in $\Lambda n$; hence $\Lambda e_{1}$ is not a primary submodule of $\Lambda$; finally, the only left ideal containing $\Lambda e_{1}$ is $\mathrm{m}_{1}$, so that $\Lambda e_{1}$ is certainly irreducible. Thus $\Lambda e_{1}$ is a tertiary submodule of $\Lambda$, but not a primary submodule $\left({ }^{4}\right)$. In $\S 6$ we will discuss conditions under which tertiaries are primary.

4. Comparison of decomposition theories. Let $C$ be an admissible category, and $\Gamma, \Omega$ two predecomposition theories on $C$. We say that $\Gamma$ is stronger than $\Omega$ if for all $(M, N) \in C, \Gamma(M / N) \supseteq \Omega(M / N)$. It is clear that if $\Gamma$ is stronger than $\Omega$, then all $\Gamma$-submodules of $M$ are also $\Omega$-submodules, and that if $(M, N)$ is $\Gamma$ finite, then it is also $\Omega$-finite.

LEMMA 4.1. Let $\Gamma$ be stronger than $\Omega$, and let $(0)$ be a $\Gamma$-submodule of $M$. Then (0) is also an $\Omega$-submodule of $M$ and $\Omega(M)=\Gamma(M)$.

Proof. If $a \in \Omega(M)$, then since $\Gamma$ is stronger than $\Omega, \mathfrak{a} \in \Gamma(M)$. Since (0) is a $\Gamma$-submodule of $M, \Gamma(M)$ consists of a single element, and hence $\Gamma(M)=\{\mathfrak{a}\}$. It follows immediately that $\Omega(M)=\{\mathfrak{a}\}$.

(3) See the remarks above following the list of basic results of the primary and tertiary theories. This is the starting point of Auslander's axiomatic development in the commutative case.

(4) Curtis [10] uses the ring $\Lambda$ of this example to show that in an arbitrary Noetherian ring, an ideal (in this case, (0)) need not be an intersection of primary ideals. 
Proposition 4.2. Let $\Gamma, \Omega$ be predecomposition theories for $C$, and suppose that $\Gamma$ is stronger than $\Omega$. If $\Gamma$ is a decomposition theory for $C$, then $\Omega(M / N)$ $=\Gamma(M / N)$ for all $\Gamma$-finite elements $(M, N) \in C$.

Proof. It suffices to take $N=(0)$. Thus, let $M$ be a $\Gamma$-finite module in $C$. Let $M_{1}, \ldots, M_{k}$ be a $\Gamma$-decomposition of (0) in $M$. The $M_{i}$ are $\Gamma$-submodules of $M$, and hence, since $\Gamma$ is stronger than $\Omega$, are also $\Omega$-submodules of $M$. By the lemma, $\Omega\left(M / M_{i}\right)=\Gamma\left(M / M_{i}\right), i=1, \ldots, k$. It follows by Proposition 1.1 that the $M_{1}, \ldots, M_{k}$ are an $\Omega$-decomposition of $(0)$ in $M$. Then by Proposition 1.2 we have:

$$
\Omega(M)=\left\{\Gamma\left(M / M_{1}\right), \ldots, \Gamma\left(M / M_{k}\right)\right\}=\Gamma(M) .
$$

Corollary 4.3. Assume that $\Lambda$ is a Noetherian ring and that $C$ is an admissible category of pairs of finitely generated modules. Let $\Gamma, \Omega$ be two predecomposition theories on $C$, and suppose that $\Gamma$ is stronger than $\Omega$. Then if $\Gamma$ is a decomposition theory, so is $\Omega$, and moreover, $\Gamma=\Omega$.

Proof. By Corollary 2.5, $\Gamma$ satisfies property $(\mathrm{P})$, and thus by 2.4 the elements in $C$ are $\Gamma$-finite. Then by the previous proposition we have $\Omega(M / N)=\Gamma(M / N)$ for all $(M, N) \in C$.

In particular, any two decomposition functors on the category of finitely generated modules which are comparable, in the sense that one is stronger than the other, must be equal. This fact could be used to give another proof that in the commutative case, the primary and tertiary theories coincide.

5. Normal decompositions. Uniqueness of the tertiary theory. Let $C$ be an admissible category for decomposition theory. A predecomposition theory $\Gamma$ on $C$ is called normal if for each $(M, N) \in C$, the elements of $\Gamma(M / N)$ (1) are annihilating ideals for $M / N$, and (2) contain the annihilator of $M / N$, i.e., if $\mathfrak{a} \in \Gamma(M / N)$, then $\mathfrak{a} \bar{M}=(0)$ for some nontrivial submodule $\bar{M}$ of $M / N$, and Ann $M / N \subseteq \mathfrak{a}$.

The following lemma is a direct abstraction of a basic result in the tertiary theory (cf. the remark following Definition 1.1 in [2]).

Lemma 5.1. Let $\Gamma$ be a normal predecomposition theory on $C$ and let $M \in C$. If $(0)$ is a $\Gamma$-submodule of $M$, with $\Gamma(M)=\{\mathfrak{a}\}$, then for any submodule $P$ of $M$,

$$
P \cap(0: \mathfrak{a})=(0) \Rightarrow P=(0),
$$

where $0: \mathfrak{a}=\{x \in M \mid \mathfrak{a} x=(0)\}$.

Proof. Let $N=0: \mathfrak{a}$. Suppose that $P \cap N=(0)$, and $P \neq(0)$. Then $\Gamma(P)$ $=\{\mathfrak{a}\}$, also, and $\mathfrak{a}$ is an annihilating ideal for $P$. Let $N^{\prime}=\{x \in P \mid \mathfrak{a} x=(0)\}$. Then $N^{\prime} \neq(0)$, and $\mathfrak{a} N^{\prime}=(0)$, Hence $N^{\prime} \subseteq N$, and so $N^{\prime} \subseteq P \cap N=(0)$. This is a contradiction. Hence $P=(0)$. 
LEMmA 5.2. Let $\mathfrak{p}_{1}, \ldots, \mathfrak{p}_{k}$ be prime ideals in $\Lambda$, and let $\mathfrak{I}$ be a left ideal contained in the union of the $\mathfrak{p}_{i}$, i.e., $\mathfrak{I} \subseteq \mathfrak{p}_{1} \cup \ldots \cup \mathfrak{p}_{k}$. Then $\mathfrak{l}$ is contained in one of them; $\mathfrak{l} \subseteq \mathfrak{p}_{i}$, for some $i$.

Proof. This lemma is well known in the commutative case. The present proof is the same as that given in [9, Vol. I, p. 215].

We may suppose that $\mathfrak{p}_{i} \notin \mathfrak{p}_{j}$ for $i \neq j$. Suppose the proposition false. Set $\mathfrak{l}_{i}=\mathfrak{I} \cap \mathfrak{p}_{1} \cap \ldots \cap \hat{\mathfrak{p}}_{i} \cap \ldots \cap \mathfrak{p}_{k}$. We may assume that $\mathfrak{l} \neq(0)$. Then $\mathfrak{l}_{i} \ddagger \mathfrak{p}_{i}$; $i=1, \ldots, k$, since, otherwise, $\left(\mathfrak{p}_{1} \cap \ldots \cap \hat{\mathfrak{p}}_{i} \cap \ldots \cap \mathfrak{p}_{k}\right) \cap \mathfrak{I} \subseteq \mathfrak{p}_{i}$, and then, since $\mathfrak{I}$ is a left ideal, $\left(\mathfrak{p}_{1} \cap \ldots \cap \hat{\mathfrak{p}}_{i} \cap \ldots \cap \mathfrak{p}_{k}\right) \cdot \mathfrak{l} \subseteq \mathfrak{p}_{i}$; the fact that $\mathfrak{p}_{i}$ is prime then gives $\mathfrak{p}_{1} \cap \ldots \cap \hat{\mathfrak{p}}_{i} \cap \ldots \cap \mathfrak{p}_{k} \subseteq \mathfrak{p}_{i}$; and this leads to a contradiction of the fact that no $\mathfrak{p}$ contains another. Choose $l_{i} \neq 0$ in $\mathfrak{I}_{i}, l_{i} \in \mathfrak{p}_{i}$. Then $l_{1}+\ldots+l_{k} \in \mathfrak{I}$, and it is easy to see that $l_{1}+\ldots+l_{k}$ is not in $\mathfrak{p}_{1} \cup \ldots \cup \mathfrak{p}_{k}$. This is a contradiction to the fact that $\mathfrak{I} \subseteq \mathfrak{p}_{1} \cup \ldots \cup \mathfrak{p}_{k}$, and proves the lemma.

LeMma 5.3. Let $\Gamma$ be a normal predecomposition theory on the admissible category $C$. Let $(0)$ be a $\Gamma$-submodule of $M \in C$, with $\Gamma(M)=\{\mathfrak{p}\}$. Then an ideal $\mathfrak{a}$ in $\Lambda$ is an annihilating ideal for $M$ if and only if $\mathfrak{a} \subseteq \mathfrak{p}$. An element $x$ in $\Lambda$ is an annihilating element for $M$ if and only if $x \in \mathfrak{p}$.

Proof. By considering the ideal generated by $x$, we see that the second part of the proposition follows from the first. If $\mathfrak{a} \subseteq \mathfrak{p}$ then by the normality of $\Gamma, \mathfrak{p}$ is an annihilating ideal for $M$ so that $\mathfrak{a}$ is, too. Conversely, suppose that $\mathfrak{a}$ is an annihilating ideal for $M$. Let $\mathfrak{a} N^{\prime}=(0), N^{\prime} \neq(0)$.

By $5.1, N^{\prime} \cap(0: \mathfrak{p}) \neq(0)$; thus $\mathfrak{a}\left(N^{\prime} \cap(0: \mathfrak{p})\right)=(0)$, and $\mathfrak{a} \subseteq \operatorname{Ann}\left(N^{\prime} \cap(0: \mathfrak{p})\right)$. Since $\Gamma\left(N^{\prime} \cap 0: \mathfrak{p}\right)=\{\mathfrak{p}\}$, and since $\Gamma$ is normal, we have $\operatorname{Ann}\left(N^{\prime} \cap 0: \mathfrak{p}\right) \subseteq \mathfrak{p}$. Hence $\mathfrak{a} \subseteq \mathfrak{p}$, and the proposition is proved.

Proposition 5.4. Let $\Gamma$ be a normal decomposition theory on an admissible category $C$. Let $(M, N) \in C$ be $\Gamma$ finite. Then the two following statements are equivalent.

(a) $\mathfrak{a} \subseteq \mathfrak{p}$ for some $\mathfrak{p} \in \Gamma(M / N)$;

(b) $\mathfrak{a}$ is an annihilating ideal for $M / N$.

If, further, the elements of $\Gamma(M / N)$ are prime ideals, then each of the preceding statements is equivalent to:

(c) a consists entirely of annihilating elements for $M / N$.

Proof. It is sufficient to assume that $N=(0)$. Let $M_{1} \cap \ldots \cap M_{k}$ be a $\Gamma$ decomposition of $(0)$ in $M$, and let $\Gamma\left(M / M_{i}\right)=\left\{\mathfrak{p}_{i}\right\}, i=1, \ldots, k$. If $\mathfrak{a} \subseteq \mathfrak{p}$, for some $\mathfrak{p} \in \Gamma(M)$, then by the normality of $\Gamma, \mathfrak{a}$ is an annihilating ideal for $M$. This proves $(a) \Rightarrow(b)$. If $\mathfrak{a}$ is an annihilating ideal for $M$, then $\mathfrak{a}$ is also an annihilating ideal for some $M / M_{i}$. Then by $5.3, \mathfrak{a} \subseteq \mathfrak{p}_{i}$. Thus (b) $\Rightarrow(\mathrm{a})$. Suppose that the elements of $\Gamma(M)$ are primes, and let $a$ be an ideal consisting entirely of annihilating elements for $M$. Let $x \in \mathfrak{a}$. Then the ideal generated by $x$ is an annihilating 
ideal for $M$, and by the equivalence of (a) and (b), we have $x \in \mathfrak{p}$, for some $\mathfrak{p} \in \Gamma(M)$. This shows that $\mathfrak{a} \subseteq \bigcup\{\mathfrak{p} \mid \mathfrak{p} \in \Gamma(M)\}$. Since $\Gamma(M)$ is a finite set of prime ideals, we conclude by 5.2 that $\mathfrak{a} \subseteq \mathfrak{p}$ for some $\mathfrak{p} \in \Gamma(M)$. Thus (c) $\Rightarrow$ (a). That (b) $\Rightarrow$ (c) is clear. The proposition is proved.

REMARK. It follows immediately that if $\Gamma$ is a normal decomposition theory, and $(M, N)$ is $\Gamma$-finite, the set of annihilating elements for $M / N$ is precisely the union, $\{\mathfrak{p} \mid \mathfrak{p} \in \Gamma(M / N)\}$, of the elements in $\Gamma(M / N)$.

Specializing to the tertiary case, we obtain the result mentioned at the beginning of $\$ 3$ :

COROLlaRY 5.5. Let $\Lambda$ be a Noetherian ring and $M$ a finitely generated $\Lambda$ module. A left ideal $\mathfrak{a}$ in $\Lambda$ is an annihilating left ideal for $M$ if and only if $a$ consists entirely of annihilating elements for $M$.

Proof. The tertiary theory is normal, and the elements of $T(M)$ are prime ideals. Then 5.4 applies.

REMARK. In [1] Lesieur and Croisot define the concept of primal left ideal. Their definition, adapted to modules, reads as follows: a submodule $N$ of $M$ is a primal submodule if, whenever $x_{1} \Lambda_{1} m_{1} \subseteq N, \ldots, x_{k} \Lambda m_{k} \subseteq N$, for elements $x_{1}, \ldots, x_{\dot{k}} \in \Lambda$ and $m_{1}, \ldots, m_{k} \in M, m_{i} \notin N, i=1, \ldots, k$, then there exists an element $m \in M, m \notin N$, such that $x_{i} \Lambda m \subseteq N, i=1, \ldots, k$. This is equivalent, in our terminology, to: if $x_{1}, \ldots, x_{k}$ are annihilating elements for $M / N$, then $\Lambda x_{1}+\ldots$ $+\Lambda x_{k}$ is an annihilating left ideal for $M / N$. Corollary 5.5 shows then that for $\Lambda$ a Noetherian ring, and $M$ finitely generated, all proper submodules of $M$ are primal.

LEMMA 5.6. Let $\Gamma, \Omega$ be two normal decomposition theories on an admissible category $C$. Suppose that $\Gamma(M / N)=\{\mathfrak{a}\}$, for an $(M, N) \in C$, and that $\Omega(M / N)$ is finite. Then $\mathfrak{a} \in \Omega(M / N)$.

Proof. By the normality of $\Gamma, \mathfrak{a}$ is an annihilating ideal for $M$. Thus by 5.4, applied to $\Omega, \mathfrak{a} \subseteq \mathfrak{p}$ for some $\mathfrak{p} \in \Omega(M / N)$. By the normality of $\Omega$, this $\mathfrak{p}$ is an annihilating ideal for $M$, and, again by 5.4 applied to $\Gamma, \mathfrak{p} \subseteq \mathfrak{a}$. Thus $\mathfrak{a}=\mathfrak{p}$, and $\mathfrak{a} \in \Omega(M / N)$.

Proposition 5.7. Let $\Gamma$ be a normal decomposition theory on an admissible category $C$; and suppose that $\Gamma$ satisfies property (P). If $\Omega$ is a normal decomposition theory on $C$, then $\Gamma(M / N) \subseteq \Omega(M / N)$ for all $\Omega$-finite $(M, N)$.

Proof. Let $(M, N)$ be $\Omega$-finite, and let $\mathfrak{a} \in \Gamma(M / N)$. By property (P) there is a nonzero submodule $M^{\prime}$ of $M$ such that $M^{\prime}$ contains $N$, and $\Gamma\left(M^{\prime} / N\right)=\{\mathfrak{a}\}$. By the preceding lemma, $\mathfrak{a} \in \Omega\left(M^{\prime} / N\right)$. Thus $\mathfrak{a} \in \Omega(M / N)$ and $\Gamma(M / N) \subseteq \Omega(M / N)$.

If we combine this last result with Proposition 4.2 we obtain a uniqueness theorem for normal decompositions. 
THEOREM 5.8. Let $\Gamma, \Omega$ be two normal decomposition theories on an admissible category $C$. If $\Gamma$ satisfies property $(\mathrm{P})$, then $\Gamma(M / N)=\Omega(M / N)$ for all $\Omega$-finite elements $(M, N)$ of $C$.

COROLlaRY 5.9. Let $\Lambda$ be a Noetherian ring and $C$ an admissible category of pairs of finitely generated $\Lambda$-modules. If $\Gamma, \Omega$ are two normal decomposition theories on $C$, then $\Gamma(M / N)=\Omega(M / N)$ for all $(M, N) \in C$, i.e., $\Gamma=\Omega$.

In view of the fact that the tertiary theory is normal, we have immediately:

COROLlaRY 5.10. Let $\Lambda$ be a Noetherian ring and $C$ an admissible category of pairs of finitely generated modules. Then the tertiary functor, $T$, is the unique normal decomposition theory on $C$.

Goldie [4, p. 127] raises the question as to whether the tertiary theory is the only one which "provides all salient features of an ideal theory" in the sense of the classical theory in commutative Noetherian rings. Corollary 5.10 shows that the answer to this question is 'yes,' at least if one grants, and this quite reasonable, that one of the salient features of the commutative theory is that it is normal.

6. Primary decompositions. The Artin-Rees property. Let $\Gamma$ be a predecomposition theory on the admissible category $C$, and let $(M, N) \in C$. We say that $N$ is a $\Gamma$-primary submodule of $M$ if (1) $N$ is a $\Gamma$-submodule of $M$, and (2) if $\Gamma(M / N)=\{\mathfrak{a}\}$, then $\mathfrak{a}^{h} M \subseteq N$ for some non-negative integer $h$. A predecomposition theory $\Gamma$ is said to be a primary predecomposition theory if each $\Gamma$-submodule of a $\Gamma$-finite module $M \in C$ is a primary submodule of $M$.

If $C$ is an admissible category, and $\Gamma$ a predecomposition theory on $C$, a module $M \in C$ is said to be an Artin-Rees module (relative to $\Gamma$ ) if $M$ is $\Gamma$-finite, and has the Artin-Rees property, i.e., for each ideal $\mathfrak{a}$ in $\Lambda$, each submodule $N$ of $M$, and each nonnegative integer $n$, there is a nonnegative integer $h(n)$ such that $\mathfrak{a}^{h(n)} M \cap N \subseteq \mathfrak{a}^{n} N\left({ }^{5}\right)$.

THEOREM 6.1. Let $\Gamma$ be a normal decomposition theory on the admissible category $C$. Suppose that whenever $M \in C$ is $\Gamma$-finite, $M / M^{\prime}$ is also $\Gamma$-finite for all submodules $M^{\prime}$ of $C$. Then a $\Gamma$-finite element $M$ of $C$ is an Artin-Rees module relative to $\Gamma$ if and only if the $\Gamma$-submodules of $M$ are $\Gamma$-primary.

Proof. Let $M \in C$ be an Artin-Rees module, and let $N$ be a $\Gamma$-submodule of $M$ with $\Gamma(M / N)=\{\mathfrak{a}\}$. Since $\Gamma$ is normal, $\mathfrak{a}$ is an annihilating ideal for $M / N$. Set

(5) This is the form in which Lesieur and Croisot state the Artin-Rees property. The more usual form, to which it may be shown to be equivalent, is: for each ideal $\mathfrak{a}$ in $\Lambda$, and each submodule $N$ of $M$, there is a non-negative integer $t$ such that $\mathfrak{a}^{n} M \cap N=\mathfrak{a}^{n-t}\left(\mathfrak{a}^{t} M \cap N\right)$, for all $n \geqq t$. The Artin-Rees theorem [11, p. 2-04] states, in our terminology, that a finitely generated module over a commutative Noetherian ring is an Artin-Rees module. 
$M^{\prime}=N: \mathfrak{a}=\{x \in M \mid \mathfrak{a} \cdot x \subseteq N\}$. Consider the submodule $\mathfrak{a} M^{\prime}$. Since $M$ is an Artin-Rees module, there is an $n$ such that $\mathfrak{a}^{n} M \cap M^{\prime} \subseteq \mathfrak{a} M^{\prime} \subseteq N$. By Lemma 4.1, then, $\mathfrak{a}^{n} M \subseteq N$. Thus $N$ is $\Gamma$-primary. Conversely, suppose that the $\Gamma$-submodules of $M$ are $\Gamma$-primary, and that $M$ is $\Gamma$-finite. Let $\mathfrak{a}$ be an ideal in $\Lambda, N$ a submodule of $M$, and $n$ a non-negative integer. Set $N^{\prime}=\mathfrak{a}^{n} N$. Then $\mathfrak{a}^{n}\left(N / N^{\prime}\right)=(0)$ so that $\mathfrak{a}^{n} \subseteq$ Ann $N / N^{\prime}$. It follows from the normality of $\Gamma$ that $\mathfrak{a} \subseteq \mathfrak{b}$ for all $\mathfrak{b} \in$ $\Gamma\left(N / N^{\prime}\right)$. By assumption $M / N^{\prime}$ is $\Gamma$-finite so that $(0)$ has a $\Gamma$-decomposition in $M / N^{\prime}$. Applying Proposition 1.6 we see that there is a submodule $R$ of $M$ such that $N^{\prime}=R \cap N$ and $\Gamma(M / R)=\Gamma\left(N / N^{\prime}\right)$. Thus for all $\mathfrak{b} \in \Gamma(M / R), \mathfrak{a}^{n} \subseteq \mathfrak{b}$. Since $N / N^{\prime}$ is $\Gamma$-finite, $M / R$ is, too. Let $M_{1}, \ldots, M_{k}$ be a $\Gamma$-decomposition of $R$ in $M$, and let $\Gamma(M / R)=\left\{\mathfrak{b}_{1}, \ldots, \mathfrak{b}_{k}\right\}$, where $\mathfrak{b}_{i}=\Gamma\left(M / M_{i}\right)$. Then $\mathfrak{a}^{n} \subseteq \mathfrak{b}_{i}$ for each $i$. Since the $M_{i}$ are $\Gamma$-submodules of $M$, the assumption that they are $\Gamma$-primary implies that $\left(\mathfrak{a}^{n}\right)^{h i} M \subseteq M_{i}$ for some $h_{i}$, each $i$. It follows that there exists an $h$ such that $\mathfrak{a}^{n h} M \subseteq M_{1} \cap \ldots \cap M_{k}=R$. Then $\mathfrak{a}^{i h} M \cap N \subseteq R \cap N=N^{\prime}=\mathfrak{a}^{n} N$, and thus $M$ is an Artin-Rees module. The theorem is proved.

If $\Lambda$ is a Noetherian ring and $C$ an admissible category of pairs of finitely generated modules, we have seen that the tertiary theory $T$ is the only normal decomposition theory on $C$. In this case the hypothesis of the theorem is automatically satisfied, since all elements of $C$ are $T$-finite. Thus we may conclude that a finitely generated module $M$ is an Artin-Rees module if and only if the tertiary submodules of $M$ are $T$-primary. Further, the following lemma characterizes the $T$-primary submodules as exactly the primary ones.

LEMMA 6.2. $N$ is a T-primary subnodule of $M$ if and only if $N$ is a primary submodule of $M$.

Proof. Suppose that $N$ is a $T$-primary submodule of $M$, and let $\mathfrak{a}$ be an annihilating ideal for $M / N$. Then if $T(M / N)=\{\mathfrak{p}\}$, we have $\mathfrak{a} \subseteq \mathfrak{p}$, and hence $\mathfrak{a}^{h} M \subseteq N$ for some $h$. Thus $\mathfrak{a}^{h} \subseteq \operatorname{Ann} M / N$ and so $\mathfrak{a} \subseteq \operatorname{rad} M / N$. Thus $N$ is a primary submodule of $M$. Conversely, suppose that $N$ is a primary submodule of $M$. Let $\mathfrak{p}=\operatorname{rad} M / N$, and let $\mathfrak{a} \in T(M / N)$. Then $\mathfrak{a}$ is a prime ideal containing Ann $M / N$, by the normality of $T$, and hence $\mathfrak{a} \supseteq \operatorname{rad} \operatorname{Ann} M / N=\mathfrak{p}$. Thus $\mathfrak{a} \supseteq \mathfrak{p}$ for all $\mathfrak{a} \in T(M / N)$. On the other hand, each $\mathfrak{a} \in T(M / N)$ is an annihilating ideal for $M / N$, and thus since $N$ is primary, $\mathfrak{a} \subseteq \mathfrak{p}$ for all $\mathfrak{a} \in T(M / N)$. Hence $T(M / N)$ $=\{\mathfrak{p}\}$, and $N$ is a tertiary submodule of $M$. Since $N$ is primary, the condition $\mathfrak{a}^{h} \subseteq$ Ann $M / N$ holds for some $h$, and we conclude that $N$ is $T$-primary.

We have as corollary the following result of Lesieur-Croisot [2, Théorème 3.3, Corollaire $]\left({ }^{6}\right)$.

(6) This remarkable result of Lesieur-Croisot was the inspiration for the analysis presented in this section. Our Theorem 6.1 is in statement and proof a direct abstraction of the LesieurCroisot theorem. It seems to be of some interest that the connection given by 6.1 between the Artin-Rees property and primary decomposition theory subsists on what seems to be a rather general level. 
COROLlaRY 6.3. Let $\Lambda$ be a Noetherian ring and $C$ an admissible category of pairs of finitely generated $\Lambda$ modules. Then $M \in C$ is an Artin-Rees module if and only if the tertiary submodules of $M$ are primary.

Thus $T$ is a primary decomposition theory (i.e., for $M \in C$, the tertiary submodules of $M$ are primary) if and only if the modules $M \in C$ are ArtinRees modules.

The following proposition summarizes several equivalent conditions for this situation.

Lemma 6.4. If $N_{1}, M_{2}$ are $T$-submodules of $M$ with $T\left(M / M_{1}\right)=T\left(M / M_{2}\right)$ $=\{\mathfrak{a}\}$, then $N=M_{1} \cap M_{2}$ is also a $T$-submodule of $M$ with $T(M / N)=\{\mathfrak{a}\}$.

Proof. We have an obvious monomorphism $0 \rightarrow M / N \rightarrow M / M_{1} \oplus M / M_{2}$, giving immediately $T(M / N) \subseteq T\left(M / M_{1} \oplus M / M_{2}\right)=T\left(M / M_{1}\right) \cup T\left(M / M_{2}\right)=\{\mathfrak{a}\}$.

Proposition 6.5. Let $\Lambda$ be a Noetherian ring, $C$ a category of pairs of finitely generated $\Lambda$-modules, and $T$ the tertiary decomposition theory on $C$. Then the irreducible submodules of an $M \in C$ are tertiary submodules, and the following statements are equivalent:

(a) if $(M, N) \in C$ and $N$ is an irreducible submodule of $M$, then $N$ is a primary submodule of $M$;

(b) for $(M, N) \in C, N$ is a finite intersection of primary submodules of $M$;

(c) $T$ is a primary decomposition theory;

(d) the elements of $C$ are Artin-Rees modules.

If, further, $\Lambda$ is an element of $C$ (considered as a left module over itself), then each of the above is equivalent to:

(e) $\Lambda$ is an Artin-Rees module.

Proof. Since $M$ is Noetherian, each submodule of $M$ may be written as a finite intersection of irreducible submodules of $M$. Since $T$ is a decomposition theory, and the elements of $C$ are $T$-finite, we see easily that irreducible submodules are tertiary submodules. These observations show that (a) $\Rightarrow(\mathrm{b})$, and also that (c) $\Rightarrow$ (a). To show that $(\mathrm{b}) \Rightarrow(\mathrm{c})$, let $(M, N) \in C$, and suppose that $N$ is a tertiary submodule of $M$. We have to show that $N$ is a primary, or equivalently, a $T$-primary submodule of $M$. Let $N=M_{1} \cap \ldots \cap M_{k}$ be a finite intersection for $N$ of primary submodules of $M$. We want to show that the set $M_{1}, \ldots, M_{k}$ contains a tertiary decomposition of $N$ in $M$. It will then follow, since $N$ is a tertiary submodule, that this decomposition consists of a single $M_{i}$, and hence that $N$ is primary in $M$. To begin with, we may assume that no proper subset of the $M_{i}$ has $N$ for its intersection. If $T\left(M / M_{i}\right) \neq T\left(M / M_{j}\right)$, for $i \neq j$, then by definition the $M_{i}$ constitute a tertiary decomposition of $N$ in $M$. Otherwise, we have for example $T\left(M / M_{1}\right)=T /\left(M / M_{2}\right)=\left\{a_{1}\right\}$, say. By Lemma $6.4, M_{1}^{\prime}=M_{1} \cap M_{2}$ is a tertiary submodule of $M$ and $T\left(M / M_{1}^{\prime}\right)=\left\{a_{1}\right\}$. Consider the intersection $N=M_{1}^{\prime}$ 
$\cap M_{3} \cap \ldots \cap M_{k}$. Repeated application of the lemma in the manner just described will yield the desired tertiary decomposition of $N$. Thus (b) $\Rightarrow$ (c). We have already seen that (c) and (d) are equivalent. To finish the proof we have only to show, assuming that $\Lambda \in C$, that (d) and (e) are equivalent. That (d) $\Rightarrow$ (e) is obvious, since $\Lambda \in C$. Suppose that $\Lambda$ is an Artin-Rees module. We show first that any finitely generated free $\Lambda$-module is an Artin-Rees module. For this it suffices to show that if $M, N$ are Artin-Rees modules, then $M \oplus N$ is, also. Let $R$ be a tertiary submodule of $M \oplus N$. Then $M \cap R$ is a tertiary submodule of $M$, by Lemma 1.3, and $N \cap R$ is a tertiary submodule of $N$. Since $M, N$ are ArtinRees modules, there exist $h, k$ such that $\mathfrak{a}^{h} M \subseteq M \cap R, \mathfrak{a}^{k} N \subseteq N \cap R$, where $\mathfrak{a}=T((M \oplus N) / R)=T(M / M \cap R)=T(N / N \cap R)$ (these equalities hold by 1.3). Then

$$
\mathfrak{a}^{\max (h, k)} \cdot(M \oplus N) \subseteq(M \cap R) \oplus(N \cap R) \subseteq R .
$$

Thus $R$ is $T$-primary, and hence primary, and it follows that $M \oplus N$ is an ArtinRees module. We have proved that finitely generated free modules are ArtinRees modules. Now let $M \in C$. Then $M$ is a quotient of a free module; suppose we have an exact sequence $0 \rightarrow K \rightarrow F \rightarrow M \rightarrow 0$ where $F$ is finitely generated and free. If $M^{\prime}$ is a tertiary submodule of $M$ there corresponds to $M^{\prime}$ a tertiary submodule $F^{\prime}$ of $F$ such that $T\left(F / F^{\prime}\right)=T\left(M / M^{\prime}\right)=\{a\}$. Since the tertiary submodules of $F$ are primary, there is an $h$ such that $\mathfrak{a}^{h} F \subseteq F^{\prime}$. Hence $\mathfrak{a}^{h} M \subseteq M^{\prime}$, and $M^{\prime}$ is $T$-primary. Hence $M^{\prime}$ is primary, and this proves that the tertiary submodules of $M$ are primary. Hence $M$ is an Artin-Rees module. Thus all $M \in C$ are Artin-Rees modules, and we conclude that $T$ is a primary decomposition theory. This proves (d) and the proposition.

Murdoch [5, p. 57] has raised the question as to when the ideals in a ring may be written as finite intersections of primary ideals. This problem is also considered by Tominaga [6] who solves it by giving a set of necessary and sufficient conditions for this to be the case. We have not specifically considered two-sided ideals in this paper, but it would not be difficult to adapt our methods to give the following alternative solution: The ideals in a Noetherian ring $\Lambda$ can be written as finite intersections of primary ideals if and only if $\Lambda$ has the Artin-Rees property for two-sided ideals, i.e., given ideals $\mathfrak{a}, \mathfrak{b}$ in $\Lambda$, and a non-negative integer $n$, there is another non-negative integer $h(n)$ such that $\mathfrak{a}^{h(n)} \cap \mathfrak{b} \subseteq \mathfrak{a}^{n} \mathfrak{b}\left({ }^{7}\right)$. We will give here the solution of the analogous problem for left ideals. Thus:

Proposition 6.6. Let $\Lambda$ be a Noetherian ring. Then the left ideals in $\Lambda$ may be written as finite intersections of primary left ideals if and only if $\Lambda$ has the Artin-Rees property, i.e., is, as left module over itself, an Artin-Rees module (relative to the tertiary functor $T$ ).

(7) All of the tertiary decomposition theory may be adapted to the case of two-sided ideals. Cf. [1, pp. 22-07, ff.]. 
Proof. Since $\Lambda$ is a Noetherian ring, it is $T$-finite, considered as an element of the category of all pairs of finitely generated $\Lambda$-modules. If the left ideals in $\Lambda$ are finite intersections of primary left ideals, then the tertiary left ideals are primary, and thus $\Lambda$ is an Artin-Rees module. Conversely, if $\Lambda$ is an ArtinRees module, 6.5 gives immediately that for any pair $(M, N)$ in the category of all pairs of finitely generated $\Lambda$-modules, $N$ is a finite intersection of primary submodules of $M$. Taking $M=\Lambda$, we conclude that any left ideal in $\Lambda$ may be written as a finite intersection of primary left ideals.

Curtis [10] defines a ring with Noetherian ideal theory as being one having the ascending chain condition on (two-sided) ideals, in which each ideal may be written as a finite intersection of primary ideals. We will say, more generally, that a Noetherian ring $\Lambda$ is a classical ring, if each submodule of a finitely generated $\Lambda$-module $M$ may be written as a finite intersection of primary submodules of $M$. Thus $\Lambda$ is a classical ring if and only if it has the Artin-Rees property. In this connection we remark that the ring $\Lambda$ defined at the end of $\S 3$ is (because of the fact that it contains nonprimary, irreducible left ideals) an example of a Noetherian ring which is not a classical ring. Curtis gives some examples of "classical" rings (in his sense), and shows that for such rings the Krull intersection theorem is valid. In the next section we will consider these matters briefly; we finish up the present section with an application of our results to commutative rings.

Part of the statement of 6.2 is that the primary submodules of an $M \in C$ are tertiary. Corollary 6.3 shows that the converse holds if and only if $M$ is an ArtinRees module. Applying this to the case of a commutative ring, we obtain, proving first that in this situation tertiary submodules are primary, a purely "idealtheoretic" proof of the so-called Artin-Rees theorem.

Lemma 6.7. Let $\Lambda$ be a commutative, Noetherian ring, and $C$ an admissible category of pairs of finitely generated modules. Then for $(M, N) \in C$, $N$ is a tertiary submodule of $M$ if and only if $N$ is a primary submodule of $M$.

Proof. We have remarked that if $N$ is a primary submodule, it is also a tertiary submodule. Conversely, suppose that $N$ is a tertiary submodule of $M$. Since $\Lambda$ is commutative, the irreducible submodules of $M$ are primary submodules $\left({ }^{8}\right)$, and hence $T$-primary by 6.2 . Thus by Proposition $6.5, T$ is a primary decomposition theory on $C$. Hence $N$, being a tertiary submodule, is $T$-primary, and thus primary.

COROLlARY 6.8. (ARTIN-REES THEOREM). Let $\Lambda$ be a commutative Noetherian ring. Then the finitely generated $\Lambda$-modules are Artin-Rees modules.

(8) This is well known in commutative ring theory. The proof may be found in [9, Vol. 1, Lemma 2, p. 209]. It is worth remarking that this is only point in the development of commutative ideal theory (at least through the basic results dealing with existence, uniqueness, etc., of primary decompositions) at which the commutativity of the ring needs to be used. 
7. Classical rings. Concluding remarks. We begin with some examples of classical rings.

The commutative Noetherian rings are, of course, classical rings. Somewhat more generally, any Noetherian ring in which all ideals are two-sided is a classical ring, for the assumption that the ideals are two-sided implies that $x \Lambda=\Lambda x$ for all $x \in \Lambda$; this fact implies that a left ideal $I$ in $\Lambda$ is primary in our sense if and only if it is primary in the usual sense, viz. $x y \in \mathfrak{l}, x \notin \mathfrak{l} \Rightarrow$ some power of $y$ is in $\mathfrak{I}$. Then the proof of $[14$, Lemma 3, p. 21] goes through without change to show that the irreducible ideals in $\Lambda$ are primary. If $\Lambda$ is a classical ring and $\Gamma$ a factor ring of $\Lambda$, then $\Gamma$ is also a classical ring. If $\Lambda$ is classical, then any full matrix ring $\Gamma$ over $\Lambda$ is a classical ring; this may be easily verified, via the Artin-Rees property, by using the fact that the (two-sided) ideals in the $n \times n$ matrix ring $\Gamma=\Lambda_{n}$ are of the form $A=\mathfrak{a}_{n}$, where $\mathfrak{a}$ is an ideal in $\Lambda$. In particular, if $\Lambda$ is a commutative Noetherian ring, the endomorphism ring of a finitely generated free $\Lambda$-module is a classical ring. This is also a special case of the following more general result.

Proposition 7.1. Let $R$ be a commutative, Noetherian ring, and $\Lambda$ a central $\left({ }^{9}\right)$ $R$-algebra, finitely generated as $R$-module. If the ideals in $\Lambda$ come from its center, i.e., if each ideal $\mathfrak{A}$ in $\Lambda$ is of the form $\mathfrak{A}=\Lambda \cdot \mathfrak{a}$, with $\mathfrak{a}$ an ideal in $R$, then $\Lambda$ is a classical ring.

Proof. Since $R$ is Noetherian, and $\Lambda$ finitely generated, $\Lambda$ is a Noetherian ring. Let $\mathfrak{X}=\Lambda \mathfrak{a}$ be an ideal in $\Lambda, \mathfrak{l}$ a left ideal in $\Lambda$, and $n$ a non-negative integer. $\Lambda$ is a finitely generated $R$-module and $\mathfrak{I}$ is an $R$-submodule of $\Lambda$. Since $R$ is a classical ring, there is, by the Artin- Rees property, an an integer $h(n)$ such that $\mathfrak{a}^{h(n)}$ $\cdot \Lambda \cap \mathfrak{l} \subseteq \mathfrak{a}^{n} \cdot \mathfrak{I}$. Then $(\Lambda \mathfrak{a})^{h(n)} \cap \mathfrak{l} \subseteq(\Lambda \mathfrak{a})^{h} \mathfrak{l}$, and $\mathfrak{A}^{h(n)} \cap \mathfrak{I} \subseteq \mathfrak{A}^{n} \mathfrak{l}$, showing that $\Lambda$ has the Artin-Rees property.

Let $\Lambda$ be a separable $R$-algebra, finitely generated as $R$-module, and let $C$ denote the center of $\Lambda$. It is known [12, Corollary 3.2] that the ideals in $\Lambda$ come from $C$. It follows, since $\Lambda$ is Noetherian, that $C$ is also a Noetherian ring, and hence, being commutative, a classical ring. Proposition 7.1 shows then that $\Lambda$ is a classical ring. We have proved:

Proposition 7.2. If $\Lambda$ is a separable, finitely generated algebra over the commutative Noetherian ring $R$, then $\Lambda$ is a classical ring.

If $M$ is a finitely generated, projective $R$-module, $R$ a commutative, Noetherian ring, then $\Lambda=\operatorname{Hom}_{R}(M, M)$ is a finitely generated, separable $R$-algebra [12, Proposition 5.1]. Thus $\Lambda$ is a classical ring. This includes the case of the full matrix rings over commutative Noetherian rings. Thus,

COROLlaRY 7.3. Let $R$ be a commutative Noetherian ring, and $M$ a finitely generated projective $R$-module. Then the ring of endomorphisms of $M$ is a classical ring.

(9) That is, $R$ is the center of $\Lambda$. 
Now let $R$ be a Dedekind ring, $\Sigma$ a central simple algebra over the quotient field of $R$, and $\Lambda$ a maximal order in $\Sigma$ over $R\left({ }^{10}\right)$. It is known [13] that if $\Sigma$ is a full matrix algebra, $\Lambda$ is the ring of endomorphisms of a finitely generated projective $R$-module. By 7.3 then, $\Lambda$ is a classical ring. However, by a direct argument, we may show that any maximal order over a Dedekind ring is a classical ring. Thus,

Proposition 7.4. Let $R$ be a Dedekind ring, $\Sigma$ a central simple algebra over the quotient field of $R$, and $\Lambda$ a maximal order in $\Sigma$ over $R$. Then $\Lambda$ is a classical ring.

Proof. Let $A$ be an ideal in $\Lambda$, and I a left ideal in $\Lambda$. For any integer $n$, we have to find an integer $h(n)$ such that $A^{h(n)} \cap I \subseteq A^{n} \cdot I$. Since $\Lambda$ is a maximal order, and $R$ is a Dedekind ring, $A$ may be written uniquely as a product of powers of prime ideals, $A=P_{1}^{a_{1}} \ldots P_{k}^{a_{k}}$. Suppose that the Artin-Rees property holds when $A$ is a power of a single prime ideal. Then we assert that it holds for $A=P_{1}^{a_{1}} \ldots P_{k}^{a_{k}}$. We illustrate the procedure for $k=2$; the same technique works for any value of $k$. Namely, given $n$, choose $h_{2}(n)$ such that: $P_{2}^{a_{2} h_{2}(n)} \cap \mathfrak{l} \subseteq P_{2}^{a_{2} \cdot{ }^{n}} \mathfrak{l}$; then choose $h_{1}(n)$ such that

$$
P_{1}^{a_{1} h_{1}(n)} \cap P_{2}^{a_{2} \cdot n} \mathfrak{I} \subseteq P_{1}^{a_{1} \cdot n} P_{2}^{a_{2} \cdot n} \mathfrak{I} .
$$

Then, putting these two steps together, we have

$$
P_{1}^{a_{1} h_{1}(n)} \cap P_{2}^{a_{2} h_{2}(n)} \cap \mathbb{I} \subseteq P_{1}^{a_{1} h_{1}(n)} \cap P_{2}^{a_{2} \cdot n} \mathfrak{I} \subseteq P_{1}^{a_{1} \cdot n} P_{2}^{a_{2} \cdot n} \mathfrak{I},
$$

and setting $h(n)=\max \left[h_{1}(n), h_{2}(n)\right]$, this gives (using $P \cap Q=P \cdot Q$ )

$$
\left(P_{1}^{a_{1}} \cdot P_{2}^{a_{2}}\right)^{h(n)} \cap \mathbb{I} \subseteq\left(P_{1}^{a_{1}} P_{2}^{a_{2}}\right)^{\mathfrak{n}} \mathfrak{I} \text {. }
$$

Thus it remains to be shown that if $A=P^{k}$ is a power of a prime, then for any $n$, and left ideal $\mathfrak{l}$, we can find $h(n)$ such that

$$
P^{k h(n)} \cap \mathfrak{I} \subseteq P^{k n} \cdot \mathfrak{I} \text {. }
$$

Set $\mathfrak{p}=P \cap R$. Since $\Lambda$ is a maximal order, $P$ is the only prime ideal in $\Lambda$ lying above $\mathfrak{p}$. Thus the representation of the ideal $\Lambda \mathfrak{p}$ as a product of primes is of the form $\Lambda \mathfrak{p}=P^{a}$, for some non-negative integer $a$, and so $(\Lambda \mathfrak{p})^{k}=P^{a k}$. Now $\Lambda$ is a finitely generated $R$-module, and since $R$ is a classical ring, there exists $h^{\prime}(n)$ such that $\mathfrak{p}^{k h^{\prime}(n)} \cdot \Lambda \cap \mathfrak{I} \subseteq \mathfrak{p}^{k n} \cdot \mathfrak{l}$. Hence $(\Lambda \mathfrak{p})^{k h^{\prime}(n)} \cap \mathfrak{I} \subseteq(\Lambda \mathfrak{p})^{k n} \mathfrak{l}$, and substituting $P^{k a}$ for $(\Lambda \mathfrak{p})^{k}$, we obtain

$$
P^{k a h^{\prime}(n)} \cap \mathfrak{I} \subseteq P^{k a \cdot n} \mathfrak{I} \subseteq P^{k n} \mathfrak{l}
$$

Setting $h(n)=a \cdot h^{\prime}(n)$, we have $P^{k h(n)} \cap \mathfrak{l} \subseteq P^{k n} \mathfrak{l}$, which is what we wanted to prove.

Goldie $[4$, Chapter 6] shows that principal ideal domains are also classical rings, in our terminology.

(10) Cf. [13] for the theory of maximal orders. 
We have seen in the example of $\$ 3$ that not all Artin rings are classical rings. Those which are have a particularly simple structure as the following characterization shows.

Proposition 7.5. Let $\Lambda$ be an Artin ring. Then $\Lambda$ is a classical ring if and only if it is a direct sum of finitely many primary $\left({ }^{11}\right)$ Artin rings.

Proof. First of all, let $S$ be a primary Artin ring. Then the radical $N$ of $S$ is the only prime ideal in $S$, and hence all left ideals in $S$ are tertiary submodules of $S$. Since $N$ is nilpotent, it is the radical of each proper two sided ideal of $S$, and it follows that all left ideals are primary submodules of $S$. Thus $S$ is a classical ring.

Any finite direct sum of primary Artin rings is then also a classical ring. Now let $\Lambda$ be a classical Artin ring. Let $(0)=\mathfrak{l}_{1} \cap \ldots \cap \mathfrak{l}_{k}$ be a primary decomposition of (0) in $\Lambda$. Let $P_{i}=\operatorname{rad} \Lambda / \mathfrak{I}_{i}, i=1, \ldots, k$, and let $Q_{i}=\operatorname{Ann} \Lambda / \mathfrak{I}_{i}, i=1, \ldots, k$. Then the $Q_{i}$ are themselves, as left ideals, primary submodules of $\Lambda$, with $(0)=$ $Q_{1} \cap \ldots \cap Q_{k}$, and $P_{i}$ is the radical of $Q_{i}$ with $P_{i} \neq P_{j}$ for $i \neq j$. Now the $P_{b}$ being maximal ideals, are co-maximal in pairs. We assert that the various powers of the $P_{i}$ are also co-maximal in pairs. For let $P_{i} \neq P_{j}$, and suppose that $P_{i}^{a}$ $+P_{j}^{b} \neq \Lambda$. Then $P_{i}^{a}+P_{j}^{b}$ is a proper ideal in $\Lambda$, and has a primary decomposition in $\Lambda$. If $P$ is a prime belonging to $P_{i}^{a}+P_{j}^{b}$ in $\Lambda$, then $P \supset P_{i}^{a}+P_{j}^{b}$, and hence by maximality, $P=P_{i}=P_{j}$, a contradiction. Since $P_{i}$ is the radical of $Q_{i}$, we may choose for each $i$ an integer $n_{i}$ such that $P_{i}^{n_{i}} \subseteq Q_{i}$. Then we have for $i \neq j$ : $Q_{i}+Q_{j}>P_{i}^{n_{i}}+P_{j}^{n_{j}}=\Lambda$, showing that the $Q_{i}$ are also co-maximal in pairs. The Chinese Remainder Theorem $\left({ }^{12}\right)$ then yields the isomorphism $\Lambda=\Lambda / Q_{1}$ $\oplus \ldots \oplus \Lambda / Q_{k}$. The rings $\Lambda / Q_{i}$ are primary, Artin rings and the proposition is proved.

Now we turn to the promised example $(\$ 2)$ of a predecomposition theory which is not a decomposition theory.

Define, on the category $C$ of finitely generated modules over the Noetherian ring $\Lambda$, the functor $S u p p$ by the prescription: Supp $M / N$ is the set of prime ideals containing Ann $M / N$. It is easy to verify that Supp is a predecomposition theory on $C$, and that it is stronger than the tertiary theory.

If Supp is a decomposition theory on $C$, then it will coincide with the tertiary theory, by Corollary 4.3. We summarize several equivalent conditions for this to be the case.

Proposition 7.6. Let $\Lambda$ be a Noetherian ring, and denote by $C$ the admissible category of pairs of finitely generated modules. The following statements are equivalent:

(11) A primary ring is one which modulo its radical is a simple Artin ring.

(12) The generalized version of this reads as follows: let $\Lambda$ be any ring, and $\mathfrak{a}_{1}, \ldots, \mathfrak{a}_{n}$ a set of ideals in $\Lambda$ which are co-maximal in pairs. If $\mathfrak{a}=\mathfrak{a}_{1} \cap \ldots \cap \mathfrak{a}_{n}$, then the natural map $\Lambda / \mathfrak{a} \rightarrow \Lambda / \mathfrak{a}_{1} \oplus \ldots \oplus \Lambda / \mathfrak{a}_{n}$ is an isomorphism onto. Cf. [4, Theorem 6.19, p. 114]. 
(a) Supp is a decomposition theory on $C$;

(b) the prime ideals in $\Lambda$ are maximal ideals, and the primes containing Ann $M / N$ are annihilating ideals for $M / N$ for all $(M, N) \in C$;

(c) $\Lambda$ is a classical ring in which the prime ideals are maximal;

(d) $\Lambda$ is a finite direct sum of classical rings each of which has a single prime ideal.

Proof. Suppose that Supp is a decomposition theory on $C$. Then it satisfies property (P); applying the latter to the submodule (0) of $\Lambda$, we see that Supp $\Lambda$ is just the set of primes in $\Lambda$, and then (P) implies that for each prime $P$, there is a left ideal $\mathfrak{I}$ such that $P$ is the only prime containing Ann $\Lambda / \mathfrak{l}$. It follows that the primes in $\Lambda$ are maximal ideals. Further, Supp coincides with the tertiary theory, by Corollary 4.3, so that in particular Supp is a normal decomposition theory. Hence the primes in Supp $M$ are annihilating ideals for $M$. This proves that (a) $\Rightarrow(b)$. Again, if Supp is a decomposition theory, and hence coincides with the tertiary theory, let $\mathfrak{I}$ be a tertiary left ideal in $\Lambda$, with $T(\Lambda / \mathfrak{l})=\{P\}$. Then Supp $\Lambda / \mathfrak{l}=\{P\}$, and hence $P$ is the only prime containing Ann $\Lambda / \mathfrak{l}$. Thus $\operatorname{rad} \Lambda / \mathfrak{l}=\{P\}$, and it follows that $\mathbb{I}$ is a primary left ideal in $\Lambda$. Thus $\Lambda$ is a classical ring; we have proved that (a) $\Rightarrow$ (c). Now assume (b). It follows easily that Supp $M \subseteq T(M)$, using the fact that the annihilating ideals for $M$ are just the ideals contained in an element of $T(M)$, and thus that Supp $M=T(M)$ for all $M$. This proves that (b) $\Rightarrow(\mathrm{a})$. To show that $(\mathrm{c}) \Rightarrow(\mathrm{b})$, let $\Lambda$ be a classical ring in which the primes are maximal. Let $(M, N) \in C$ and let $P$ be a prime ideal containing Ann $M / N$. Then $P$ contains $\operatorname{rad} M / N$. If $P_{1}, \ldots, P_{n}$ are the primes belonging to $N$ in $M$, then $\operatorname{rad} M / N=P_{1} \cap \ldots \cap P_{n}$, $\Lambda$ being a classical ring, and thus $P$ contains one of the $P_{i}$. By the maximality of $P_{i}$, this gives $P=P_{i}$, so that $P$ belongs to $N$ in $M\left({ }^{13}\right)$. Hence $P$ is an annihilating ideal for $M$. This proves (b). Finally, if $\Lambda$ is a classical ring in which the primes are maximal, the proof of 7.5 shows that $\Lambda$ may be written as a finite direct sum of classical rings, each of which has a single prime ideal. Hence $(c) \Rightarrow(d)$. That $(d) \Rightarrow(c)$ is easy. The proposition is proved.

The equivalence of (a) and (c), then, shows that the example presented at the end of $\S 3$ is a ring in which Supp is not a decomposition theory. If $\Lambda$ is a commutative Noetherian ring, the condition that Supp is a decomposition theory, then, is equivalent to the condition that the primes are maximal, and hence, using the well-known fact that a Noetherian ring in which the primes are maximal has minimum condition, to the condition that $\Lambda$ is an Artin ring. I do not know whether this same equivalence holds in general.

The equivalence of (b) and (c), together with Proposition 7.5 yields the following result, which as far as I am aware has not appeared in the literature.

(13) This argument can be adapted to prove the following, a result well known in the commutative case: if $\Lambda$ is a classical ring, the minimal primes containing Ann $M / N$ are annihilating ideals for $M / N$. 
Proposition 7.7. An Artin ring $\Lambda$ is a direct sum of primary rings if and only if the primes containing Ann $M$ are annihilating ideals for $M$, for all finitely generated $\Lambda$-modules $M$.

We finish the paper by showing that in a classical ring, the Krull "intersection theorem holds $\left({ }^{14}\right)$.

Proposition. 7.8. Let $\Lambda$ be a classical ring and $\mathfrak{a}$ an ideal in $\Lambda$. Set $\Delta=\bigcap_{n} \mathfrak{a}^{n}$. Then $\mathfrak{a} \Delta=\Delta$. Thus if $\mathfrak{a}$ is contained in the radical of $\Lambda$ we have $\bigcap_{n} \mathfrak{a}^{n}=(0)$.

Proof. By the Artin-Rees property, there exists $h(1)$ such that $\mathfrak{a}^{h(1)} \cap \Delta \subseteq \mathfrak{a} \cdot \Delta$. Then $\Delta=\mathfrak{a}^{h(1)} \cap \Delta \subseteq \mathfrak{a} \cdot \Delta \subseteq \Delta$, and $\Delta=\mathfrak{a} \Delta$. If $\mathfrak{a}$ is contained in the radical of $\Lambda$, it is well known that this last equality implies that $\Delta=(0)$.

\section{BIBLIOGRAPHY}

1. R. Croisot, Séminaire P. Dubreil et C. Pisot, 1956/57, Algebre et Théories des Nombres, Exposé 22.

2. L. Lesieur and R. Croisot, Extension au cas non commutatif d'un théorème de Krull et d'un lemme d'Artin-Rees, J. Reine Angew. Math. 204 (1960), 216-220.

3. L. Lesieur, Séminaire P. Dubreil-Jacotin et C. Pisot, 1957-58, Algebre et Théorie des Nombres, Exposé 14.

4. A. W. Goldie, Rings with maximum condition, Lecture Notes, Yale University.

5. D. C. Murdoch, Contributions to non-commutative ideal theory, Canad. J. Math. 4 (1952), 43-57.

6. $\mathrm{H}$. Tominaga, On primary ideal decompositions in non-commutative rings, Math. J. Okayama Univ. 3 (1953), 37-46.

7. A. Grothendieck, Eléments de geometrie algébrique, Chapter I, Publ. Math. Inst. Haute Études Sci., No. 4, 1960.

8. N. Jacobson, Structure of rings. Amer. Math. Soc. Colloq. Publ. Vol. 37, Amer. Math. Soc., Providence, R. I., 1956.

9. O. Zariski and P. Samuel, Commutative algebra, Van Nostrand, Princeton, N. J., 1958.

10. C. Curtis, Additive ideal theory in general rings, Amer. J. Math. 74 (1952), 687-700.

11. H. Cartan et C. Chevalley, Séminaire de l'École Normale Supérieure, 1955-56, Géometrie Algébrique.

12. M. Auslander and $\mathrm{O}$. Goldman, The Brauer group of a commutative ring, Trans. Amer. Math. Soc. 97 (1960), 367-409.

13. - Trans. Amer. Math. Soc. 97 (1960), 1-24.

14. D. G. Northcott, Ideal theory, Cambridge Tracts No. 42, Cambridge Univ. Press, Cambridge, 1953.

Parke Mathematical Laboratories, Inc., CARLisle, MassachusetTs

BRANDEIS UNIVERSITY, Waltham, MassachusetTs

(14) See [2] for a "tertiary" generalization of this, and also for an example of a ring, necessarily not a classical ring, by our 7.8 , in which the equality $\Delta=\mathfrak{a} \Delta$ fails to hold. 